(2) Open Access Full Text Article

\title{
Design, synthesis, and anticancer activity of novel berberine derivatives prepared via CuAAC "click" chemistry as potential anticancer agents
}

This article was published in the following Dove Press journal:

Drug Design, Development and Therapy

6 August 2014

Number of times this article has been viewed

\author{
Xin Jin ${ }^{1,2, *}$ \\ Tian-Hua Yan $^{3, *}$ \\ Lan Yan' \\ Qian $\mathrm{Li}^{4}$ \\ Rui-Lian Wang' \\ Zhen-Lin $\mathrm{Hu}^{\prime}$ \\ Yuan-Ying Jiang' \\ Qing-Yan Sun' \\ Yong-Bing $\mathrm{CaO}^{\prime}$
}

'School of Pharmacy, Second

Military Medical University,

Shanghai, People's Republic of

China; ${ }^{2}$ School of Pharmacy, Fujian

University of Traditional Chinese

Medicine, Fuzhou, People's Republic

of China; ${ }^{3}$ Department of

Pharmacology, School of Pharmacy,

China Pharmaceutical University,

Nanjing, People's Republic of China;

${ }^{4}$ Diakite Biological Technology Co.,

Ltd, Shanghai, People's Republic of

China

*These authors contributed equally to this work
Correspondence: Qing-Yan Sun,

Yong-Bing $\mathrm{CaO}$

School of Pharmacy, Second Military

Medical University, Shanghai 200433,

People's Republic of China

Tel +862 2। $8 \mid 87$ I 275

$\mathrm{Fax}+86218187 \quad 275$

Email sqy_2000@163.com, ybcao@vip. sina.com

\begin{abstract}
A series of novel derivatives of phenyl-substituted berberine triazolyls has been designed and synthesized via copper-catalyzed azide-alkyne cycloaddition click chemistry in an attempt to develop antitumor agents. All of the compounds were evaluated for anticancer activity against a panel of three human cancer cell lines, including MCF-7 (breast), SW-1990 (pancreatic), and SMMC-7721 (liver) and the noncancerous human umbilical vein endothelial cell (HUVEC) cell lines. The results indicated that most of the compounds displayed notable anticancer activities against the MCF-7 cells compared with berberine. Among these derivatives, compound 16 showed the most potent inhibitory activity against the SW-1990 and SMMC-7721 cell lines, with half-maximal inhibitory concentration $\left(\mathrm{IC}_{50}\right)$ values of $8.54 \pm 1.97 \mu \mathrm{M}$ and $11.87 \pm 1.83 \mu \mathrm{M}$, respectively. Compound 36 exhibited the most potent inhibitory activity against the MCF-7 cell line, with an $\mathrm{IC}_{50}$ value of $12.57 \pm 1.96 \mu \mathrm{M}$. Compound 16 and compound 36 exhibited low cytotoxicity in the HUVEC cell line, with $\mathrm{IC}_{50}$ values of $25.49 \pm 3.24 \mu \mathrm{M}$ and $30.47 \pm 3.47 \mu \mathrm{M}$. Furthermore, compounds 14, 15, 16, 17, 18, 32, and 36 exhibited much better selectivity than berberine toward the normal cell line HUVEC.
\end{abstract}

Keywords: berberine, anticancer, click chemistry, structure-activity relationship

\section{Introduction}

In the past several decades, researchers have struggled to find effective clinical approaches for the treatment of cancer and have searched for novel anticancer agents..$^{1-3}$ Berberine, an isoquinoline alkaloid isolated from the roots and stem bark of the Berberis species, is widely used as a traditional medicine for treating diarrhea and gastrointestinal disorders. ${ }^{4}$ In the past several years, berberine has shown a wide range of biochemical and pharmacological activities. ${ }^{5-11}$ Research on the anticancer activity of berberine in particular has received widespread attention and has achieved fairly good results. ${ }^{12-19}$ However, berberine was poorly absorbed in the intestines and thus showed a low inhibitory effect on cancer cell growth, which seriously affected the application prospects of berberine as an anticancer drug. ${ }^{20-21}$

The concept of "click" chemistry was introduced in 2001. The catchy term "click" refers to the facile, efficient, selective, and versatile chemical transformations that lead to a single reaction product. ${ }^{22,23}$ Although different chemical reactions (eg, cycloadditions, nucleophilic substitutions, additions to carbon-carbon double bonds) can be considered to be of the "click" type, copper(I)-catalyzed azide-alkyne cycloaddition $(\mathrm{CuAAC})$ is regarded as a prime example of "click" chemistry. ${ }^{24,25}$ This reaction is regioselective, chemoselective, and moreover can be performed in aqueous medium at room or physiological temperature. Several recent reports have confirmed that $\mathrm{CuAAC}$ 
is a very useful method in bioapplications as diverse as drug discovery, drug delivery, and gene therapy. ${ }^{26-32}$ Thus, CuAAC has become a very popular tool in drug research.

To enhance the inhibitory function of berberine, the functionalized benzyl-modified berberine derivative compounds 4-36 were synthesized via CuAAC "click" chemistry from berberrubine and relevant azides and screened for cytotoxicity in vitro in human MCF-7 breast adenocarcinoma cells, human SW-1990 pancreatic carcinoma cells, and human SMMC-7721 liver carcinoma cells. This study is presented in two parts for clarity, the synthesis of new triazolyl berberine derivatives and the results of testing their anticancer properties. Several types of functional groups, specifically triazolyl berberine derivatives, were investigated to determine the structure-activity relationship.

\section{Materials and methods}

\section{Synthesis and purification}

\section{of triazolyl berberine derivatives}

In this study, 33 triazolyl berberine derivatives were synthesized. This is the first report of any of them. The initial synthesis of the triazolyl berberine derivatives is illustrated in Figure 1. First, structural modifications at berberine C-9 (compound 1) can be envisaged after converting the methoxy group to a hydroxyl to obtain berberrubine (compound 2). Stirring compound 1 with dimethylformamide (DMF) at $190^{\circ} \mathrm{C}$ yielded berberrubine. ${ }^{33}$ Second, compound 3 was synthesized with an overall yield of $60 \%$ from berberrubine and propargyl bromide by a nucleophilic substitution reaction. Azides were prepared from their corresponding benzyl halides by diazotization in acidic conditions followed by displacement with sodium azide, with good to excellent yield (approximately $85 \%$ yield). Reaction of compound 3 with aromatic azides in the presence of $\mathrm{CuSO}_{4} \cdot 5 \mathrm{H}_{2} \mathrm{O}$ and sodium ascorbate in DMF resulted in the derivatives 4-36 in excellent yields. The structures of the synthesized target compounds were elucidated by ${ }^{1} \mathrm{H}$ nuclear magnetic resonance (NMR), ${ }^{13} \mathrm{C}$ NMR, and mass spectrometry (MS). By employing the above reaction condi- tions, a series of triazolyl berberine derivatives that have varied substitutions on the aromatic ring were synthesized from a range of aromatic azides. All spectral data were in accordance with the assumed structures.

\section{Chemistry}

${ }^{1} \mathrm{H}$ NMR and ${ }^{13} \mathrm{C}$ NMR spectra were recorded using tetramethylsilane (TMS) as the internal standard in dimethyl sulfoxide (DMSO)- $d_{6}$ with a Bruker BioSpin $\mathrm{GmbH}$ (Billerica, MA, USA) spectrometer at $300 \mathrm{MHz}$ and $75 \mathrm{MHz}$, respectively. The chemical shifts are reported in ppm relative to TMS as the internal standard, and coupling constants were measured in Hz. Electrospray ionization (ESI)-MS was recorded on an Agilent $1100 \mathrm{LC} / \mathrm{MSD}$ (70 eV) spectrometer (Agilent Technologies, Santa Clara, CA, USA). High-resoution mass spectrometry was recorded on a Waters Micromass $^{\circledR}$ Q-Tof micro ${ }^{\mathrm{TM}}$ mass spectrometer (Milford, MA, USA). All other reagents and starting materials were purchased and used as received (Sigma-Aldrich Co, St Louis, MO, USA; Tokyo Chemical Industry Co, Ltd, Tokyo, Japan; Adamas Reagent, Ltd, Shanghai, China). Reactions were monitored by analytical thin-layer chromatography (TLC) using silica gel 60 F254 plates, and spots were visualized by ultraviolet light irradiation $(254 \mathrm{~nm})$. Flash column chromatography was performed with silica gel (200-300 mesh). All reagents were purchased from Sigma-Aldrich $\mathrm{Co}$, (St Louis, MO, USA). All solvents and reagents were analytical pure, and no further purification was needed. All starting materials were commercially available.

To a solution of $\mathrm{NaN}_{3}(50 \mathrm{mg}, 0.77 \mathrm{mmol})$ in DMF $(10 \mathrm{~mL})$, a benzyl compound $(0.6 \mathrm{mmol})$ was added, and the reaction mixture was sonicated at $35^{\circ} \mathrm{C}$ until completion, monitored by TLC. Compound 3 (160 mg, $0.4 \mathrm{mmol}), \mathrm{CuSO}_{4} \cdot 5 \mathrm{H}_{2} \mathrm{O}(30 \mathrm{mg}, 0.12 \mathrm{mmol})$ and sodium ascorbate (50 mg, $0.25 \mathrm{mmol}$ ) were added to this mixture. The mixture was stirred at room temperature for 3 hours, filtered and then evaporated under vacuum, while being monitored by TLC. The crude mixture was extracted with

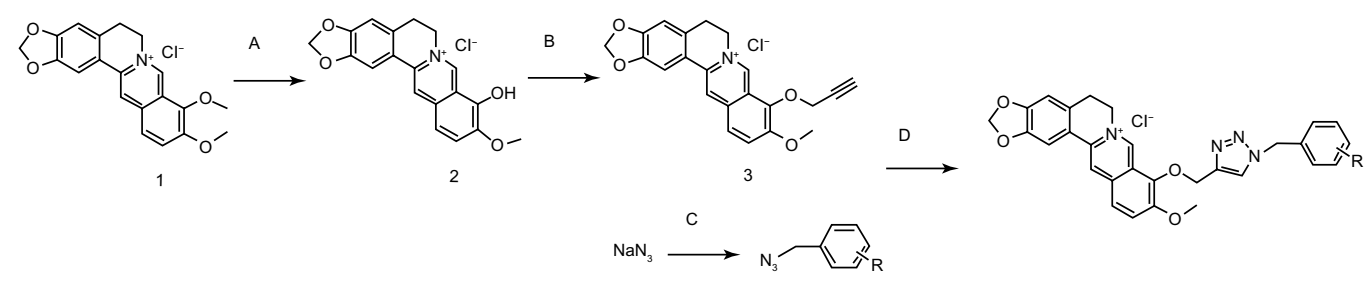

Figure I General route for synthesis of triazolyl berberine derivatives.

Notes: Reagents and conditions: (A) $190^{\circ} \mathrm{C}, 20-30 \mathrm{mmHg}, 15$ minutes; (B) $\mathrm{BrCH}_{2} \mathrm{C} \equiv \mathrm{CH}, \mathrm{CH}_{3} \mathrm{CN}$, reflux, 2.5 hours; (C) DMF, 3 hours; (D) $\mathrm{CuSO} \cdot 5 \mathrm{H}_{2} \mathrm{O}$, sodium ascorbate, DMF, $50^{\circ} \mathrm{C}$.

Abbreviation: DMF, dimethylformamide. 
dichloromethane $(3 \times 40 \mathrm{~mL})$, and the combined organic layer was dried over sodium sulfate and purified through column chromatography to give pure compounds 4-36 at $50 \%-90 \%$ yields.

\section{Synthesis of berberrubine (compound 2)}

Commercially available berberine (compound 1, $1.1 \mathrm{~g}$ ) chloride was heated at $190^{\circ} \mathrm{C}$ in a vacuum oven under reduced pressure $(20-30 \mathrm{mmHg}$ ) for 1-2 hours to obtain berberrubine (compound 2, $2.885 \mathrm{mg}$ ) in 90\% yield. Dark red solid; yield $88 \%{ }^{1} \mathrm{H}$ NMR (300 MHz, DMSO): $\delta 9.19$ (s, 1H), $7.90(\mathrm{~s}, 1 \mathrm{H}), 7.45(\mathrm{~s}, 1 \mathrm{H}), 7.34(\mathrm{~s}, 1 \mathrm{H}), 6.80(\mathrm{~s}, 2 \mathrm{H})$, $6.00(\mathrm{~s}, 2 \mathrm{H}), 4.85(\mathrm{~s}, 2 \mathrm{H}), 3.83(\mathrm{~s}, 3 \mathrm{H}), 3.09(\mathrm{~s}, 2 \mathrm{H})$; ${ }^{13} \mathrm{C}$ NMR (DMSO): $\delta 162.7,149.6,149.4,148.0,145.8$, $134.0,132.2,129.2,122.5,121.4,120.1,118.2,107.7$, 106.9, 104.3, 101.8, 55.2, 54.1, 27.5; high-resolution (HR)ESI-MS calculated for $\mathrm{C}_{19} \mathrm{H}_{16} \mathrm{NO}_{4}^{+}[\mathrm{M}+1-\mathrm{Cl}]^{+} 323.1079$, found 323.1078 .

\section{Synthesis of 9-O-(propyne) berberine chloride (compound 3)}

To a solution of compound $2(540 \mathrm{mg}, 1 \mathrm{mmol})$ in acetonitrile, propargyl bromide $(240 \mathrm{mg}, 1.2 \mathrm{mmol}$ ) was added and the reaction mixture was stirred at $75^{\circ} \mathrm{C}$ for 4 hours. Reaction was monitored by TLC and the crude product was subjected to column chromatography to give pure compound 3 (350 mg, $65 \%$ yield). Yellow solid, ${ }^{1} \mathrm{H}$ NMR (300 MHz, DMSO): $\delta 9.88(\mathrm{~s}, 1 \mathrm{H}), 8.97$ (d, J=7.8 Hz, 1H), 8.22 (t, J=8.8 Hz, 1H), 8.03 (dd, J=14.8, 9.1 Hz, 1H), 7.80 (s, 1H), 7.09 (s, 1H), $6.18(\mathrm{~s}, 2 \mathrm{H}), 5.09$ (d, J=2.4 Hz, 2H), 4.96 (t, J=6.1 Hz, 2H), 4.09 (d, J=5.6 Hz, 3H), 3.62 (t, J=2.4 Hz, 1H), 3.21 (t, J=6.1 Hz, 2H); ${ }^{13} \mathrm{C}$ NMR (75 MHz, DMSO): $\delta$ 151.20, $150.38,148.19,145.82,141.19,138.12,133.42,131.22$, $127.03,124.76,122.58,120.90,120.79,108.92,105.96$, 102.60, 80.30, 79.26, 61.44, 57.65, 55.79, 26.82; HR-ESI-MS calculated for $\mathrm{C}_{22} \mathrm{H}_{18} \mathrm{NO}_{4}^{+}[\mathrm{M}+1-\mathrm{Cl}]^{+} 361.1235$, found 361.1239 .

\section{General procedure for the synthesis of compounds 4-36}

To a solution of $\mathrm{NaN}_{3}(50 \mathrm{mg}, 0.77 \mathrm{mmol})$ in DMF $(10 \mathrm{~mL})$, compound of benzyl $(0.6 \mathrm{mmol})$ was added and the reaction mixture was sonicated at $35^{\circ} \mathrm{C}$ until completion, monitored by TLC. To this mixture, compound 3 (160 mg, $0.4 \mathrm{mmol}$ ), $\mathrm{CuSO}_{4} \cdot 5 \mathrm{H}_{2} \mathrm{O}(30 \mathrm{mg}, 0.12 \mathrm{mmol})$ and sodium ascorbate (50 mg, $0.25 \mathrm{mmol}$ ) were added. The mixture was stirred at room temperature for 3 hours, filtered, and then evaporated under vacuum, monitored by TLC. The crude mixture was extracted with dichloromethane $(3 \times 40 \mathrm{~mL})$ and the combined organic layer was dried over sodium sulfate and purified through column chromatography to give pure compounds 4-36 in 50\%-90\% yield.

\section{9-O-[4-ethyl-(I-benzyl)-IH-I,2,3-triazole]} berberine chloride (compound 4)

Yellow solid, yield 74\%; ${ }^{1} \mathrm{H}$ NMR (300 MHz, DMSO): $\delta 9.64(\mathrm{~s}, 1 \mathrm{H}), 8.94$ (d, J=15.5 Hz, 1H), 8.38 (s, 1H), 8.21 (t, J=9.3 Hz, 1H), 8.03 (t, J=12.0 Hz, 1H), 7.79 (s, 1H), 7.19 (ddd, J=38.5, 14.7, 7.5 Hz, 6H), 6.18 (s, 2H), 5.57 (s, 2H), $5.46(\mathrm{~s}, 2 \mathrm{H}), 4.80(\mathrm{~s}, 2 \mathrm{H}), 4.06(\mathrm{~s}, 3 \mathrm{H}), 3.11(\mathrm{~s}, 2 \mathrm{H}) ;{ }^{13} \mathrm{C} \mathrm{NMR}$ (75 MHz, DMSO): $\delta 151.49,150.38,148.22,145.65,143.07$, $141.82,137.89,136.48,133.36,131.11,129.17,128.51$, $128.07,127.03,126.45,125.94,124.52,122.50,120.87$, $120.78,115.67,108.93,105.92,102.61,66.82,57.67$, 55.78, 53.24, 26.81; HR-ESI-MS calculated for $\mathrm{C}_{29} \mathrm{H}_{25} \mathrm{~N}_{4} \mathrm{O}_{4}^{+}$ $[\mathrm{M}+1-\mathrm{Cl}]^{+}$494.1903, found 494.1908.

\section{9-O-[I-(2-fluorobenzyl)-4-ethyl-IH-I, 2, 3-triazole] berberine chloride (compound 5)}

Yellow solid, yield 70\%; ${ }^{1} \mathrm{H}$ NMR (300 MHz, DMSO): $\delta 9.66(\mathrm{~s}, 1 \mathrm{H}), 8.92(\mathrm{~s}, 1 \mathrm{H}), 8.33(\mathrm{~s}, 1 \mathrm{H}), 8.20(\mathrm{~d}, \mathrm{~J}=9.1$ $\mathrm{Hz}, 1 \mathrm{H}), 8.00(\mathrm{~d}, \mathrm{~J}=9.2 \mathrm{~Hz}, 1 \mathrm{H}), 7.80(\mathrm{~s}, 1 \mathrm{H}), 7.33$ (d, J=6.9 Hz, 1H), 7.16 (t, J=9.3 Hz, 3H), 7.09 (s, 1H), 6.18 (s, 2H), $5.63(\mathrm{~s}, 2 \mathrm{H}), 5.46(\mathrm{~s}, 2 \mathrm{H}), 4.83(\mathrm{~s}, 2 \mathrm{H}), 4.06(\mathrm{~s}, 3 \mathrm{H})$, 3.14 (s, 2H); ${ }^{13} \mathrm{C}$ NMR (75 MHz, DMSO): $\delta 158.36,150.94$, $149.86,147.71,145.26,142.46,141.50,137.37,133.01$, $130.74,130.59,130.50,126.50,125.50,124.84,123.98$, $121.95,120.46,120.25,115.77,115.47,108.42,105.39$, 102.10, 66.31, 56.99, 55.42, 47.02, 26.38; HR-ESI-MS calculated for $\mathrm{C}_{29} \mathrm{H}_{24} \mathrm{BrN}_{4} \mathrm{O}_{4}^{+}[\mathrm{M}+1-\mathrm{Cl}]^{+} 572.0980$, found 572.0986 .

\section{9-O-[I-(3-fluorobenzyl)-4-ethyl- IH-I, 2, 3-triazole] berberine chloride (compound 6)}

Yellow solid, yield 63\%; ${ }^{1} \mathrm{H}$ NMR (300 MHz, DMSO): $\delta 9.69$ (s, 1H), 8.93 (s, 1H), 8.47 (s, 1H), 8.20 (d, J=9.2 Hz, $1 \mathrm{H}), 8.00$ (d, J=9.0 Hz, 1H), $7.78(\mathrm{~s}, 1 \mathrm{H}), 7.34$ (dd, J=14.2, $7.5 \mathrm{~Hz}, 1 \mathrm{H}), 7.03$ (dt, J=23.2, $6.5 \mathrm{~Hz}, 4 \mathrm{H}), 6.18(\mathrm{~s}, 2 \mathrm{H})$, $5.62(\mathrm{~s}, 2 \mathrm{H}), 5.47(\mathrm{~s}, 2 \mathrm{H}), 4.85(\mathrm{~s}, 2 \mathrm{H}), 4.07$ (s, 3H), 3.13 (s, 2H); ${ }^{13} \mathrm{C}$ NMR (75 MHz, DMSO): $\delta$ 150.96, 149.93, $147.78,145.38,142.65,141.46,137.47,132.99,130.90$, $130.73,130.58,126.66,125.63,124.02,123.78,122.00$, $120.34,120.23,115.13,114.83,114.49,108.39,105.52$, 102.16, 66.59, 57.01, 55.51, 52.20, 26.39; HR-ESI-MS calculated for $\mathrm{C}_{29} \mathrm{H}_{24} \mathrm{BrN}_{4} \mathrm{O}_{4}+[\mathrm{M}+1-\mathrm{Cl}]^{+} 572.0980$, found 572.0983 . 
9-O-[I-(4-fluorobenzyl)-4-ethyl-I H-I, 2, 3-triazole] berberine chloride (compound 7)

Yellow solid, yield 65\%; ${ }^{1} \mathrm{H}$ NMR (300 MHz, DMSO): $\delta 9.69(\mathrm{~s}, 1 \mathrm{H}), 8.95(\mathrm{~s}, 1 \mathrm{H}), 8.41(\mathrm{~s}, 1 \mathrm{H}), 8.24(\mathrm{~d}, \mathrm{~J}=9.2 \mathrm{~Hz}, 1 \mathrm{H})$, $8.05(\mathrm{~d}, \mathrm{~J}=9.1 \mathrm{~Hz}, 1 \mathrm{H}), 7.82(\mathrm{~s}, 1 \mathrm{H}), 7.35-7.03(\mathrm{~m}, 5 \mathrm{H})$, $6.21(\mathrm{~s}, 2 \mathrm{H}), 5.60(\mathrm{~s}, 2 \mathrm{H}), 5.49(\mathrm{~s}, 2 \mathrm{H}), 4.86(\mathrm{~s}, 2 \mathrm{H})$, 4.10 (s, 3H), 3.16 (s, 2H); ${ }^{13} \mathrm{C}$ NMR (75 MHz, DMSO): $\delta 151.03,149.83,147.84,145.22,142.66,141.58,137.44$, $132.75,132.44,130.93,130.07,129.96,127.01,125.42$, $124.54,124.28,122.24,120.44,120.31,115.73,115.44$, 108.49, 105.53, 102.46, 66.78, 57.08, 55.64, 52.51, 26.74; HR-ESI-MS calculated for $\mathrm{C}_{29} \mathrm{H}_{24} \mathrm{BrN}_{4} \mathrm{O}_{4}^{+}[\mathrm{M}+1-\mathrm{Cl}]^{+}$ 572.0980 , found 572.0985 .

\section{9-O-[I-(2-chlorobenzyl)-4-ethyl-IH-I, 2, 3-triazole]} berberine chloride (compound 8)

Yellow solid, yield 59\%; ${ }^{1} \mathrm{H}$ NMR (300 MHz, DMSO): $\delta 9.65(\mathrm{~s}, 1 \mathrm{H}), 8.91(\mathrm{~s}, 1 \mathrm{H}), 8.40(\mathrm{~s}, 1 \mathrm{H}), 8.17-8.09(\mathrm{~m}, 1 \mathrm{H})$, $8.00(\mathrm{~d}, \mathrm{~J}=9.4 \mathrm{~Hz}, 1 \mathrm{H}), 7.75(\mathrm{~s}, 1 \mathrm{H}), 7.38(\mathrm{~d}, \mathrm{~J}=7.5 \mathrm{~Hz}, 1 \mathrm{H})$, 7.29-7.21 (m, 2H), $7.05(\mathrm{~s}, 2 \mathrm{H}), 6.15(\mathrm{~s}, 2 \mathrm{H}), 5.64(\mathrm{~s}, 2 \mathrm{H})$, 5.45 (s, 2H), 4.82 (s, 2H), 4.04 (s, 3H), 3.10 (s, 2H); ${ }^{13} \mathrm{C} \mathrm{NMR}$ (75 MHz, DMSO): $\delta 151.13,149.98,147.83,145.29,142.46$, $141.28,137.44,133.22,133.01,132.72,130.72,130.58$, $130.39,129.70,127.79,126.54,126.18,124.29,122.15$, $120.48,108.59,105.60,102.26,66.25,57.19,55.50,50.77$, 34.21, 26.47; HR-ESI-MS calculated for $\mathrm{C}_{29} \mathrm{H}_{24} \mathrm{ClN}_{4} \mathrm{O}_{4}^{+}$ $[\mathrm{M}+1-\mathrm{Cl}]^{+} 528.1486$, found 528.1490 .

\section{9-O-[I-(3-chlorobenzyl)-4-ethyl-I H-I, 2, 3-triazole]} berberine chloride (compound 9)

Yellow solid, yield 68\%; ${ }^{1} \mathrm{H}$ NMR (300 MHz, DMSO): $\delta 9.67(\mathrm{~s}, 1 \mathrm{H}), 8.93(\mathrm{~s}, 1 \mathrm{H}), 8.20$ (dd, J=18.4, $12.4 \mathrm{~Hz}, 2 \mathrm{H})$, $8.00(\mathrm{~d}, \mathrm{~J}=6.1 \mathrm{~Hz}, 1 \mathrm{H}), 7.79(\mathrm{~s}, 1 \mathrm{H}), 7.09$ (dd, J=7.3, $3.1 \mathrm{~Hz}$, $4 \mathrm{H}), 6.85(\mathrm{~s}, 1 \mathrm{H}), 6.18(\mathrm{~s}, 2 \mathrm{H}), 5.56(\mathrm{~s}, 2 \mathrm{H}), 5.47(\mathrm{~s}, 2 \mathrm{H})$, $4.80(\mathrm{~s}, 2 \mathrm{H}), 4.06$ (d, J=2.9 Hz, 3H), $3.11(\mathrm{~s}, 2 \mathrm{H}) ;{ }^{13} \mathrm{C} \mathrm{NMR}$ (75 MHz, DMSO): $\delta 150.97,149.87,147.71,145.18,142.39$, $141.22,137.36,132.83,130.59,130.46,130.33,126.12$, $125.47,124.07,123.97,122.01,120.36,120.31,115.33$, 114.63, 114.69, 108.42, 105.40, 102.10, 66.22, 57.00, 55.25, 52.94, 26.29; HR-ESI-MS calculated for $\mathrm{C}_{29} \mathrm{H}_{24} \mathrm{ClN}_{4} \mathrm{O}_{4}^{+}$ $[\mathrm{M}+1-\mathrm{Cl}]^{+} 528.1486$, found 528.1492 .

\section{9-O-[I-(4-chlorobenzyl)-4-ethyl-I H-I, 2, 3-triazole]} berberine chloride (compound 10 )

Yellow solid, yield 62\%; ${ }^{1} \mathrm{H}$ NMR (300 MHz, DMSO): $\delta 9.65(\mathrm{~s}, 1 \mathrm{H}), 8.91(\mathrm{~s}, 1 \mathrm{H}), 8.41(\mathrm{~s}, 1 \mathrm{H}), 8.20$ (d, J=9.2 Hz, $1 \mathrm{H}), 8.01(\mathrm{~d}, \mathrm{~J}=9.1 \mathrm{~Hz}, 1 \mathrm{H}), 7.77(\mathrm{~s}, 1 \mathrm{H}), 7.31(\mathrm{t}, \mathrm{J}=14.0 \mathrm{~Hz}$, 2H), 7.15 (d, J=8.4 Hz, 2H), 7.07 (s, 1H), 6.17 (s, 2H),
5.55 (d, J=19.2 Hz, 2H), 5.46 (s, 2H), 4.82 (s, 2H), 4.07 (s, 3H), 3.10 (d, J=6.1 Hz, 2H); ${ }^{13} \mathrm{C}$ NMR (75 MHz, DMSO): $\delta$ 151.01, 149.93, 147.77, 145.18, 142.64, 141.33, 137.41, $134.99,132.88,130.72,130.62,129.55,128.69,126.55$, $125.58,124.07,123.94,122.01,120.36,120.27,114.57$, 108.47, 105.49, 102.13, 66.31, 57.08, 55.34, 51.95, 26.35; HR-ESI-MS calculated for $\mathrm{C}_{29} \mathrm{H}_{24} \mathrm{ClN}_{4} \mathrm{O}_{4}^{+}[\mathrm{M}+1-\mathrm{Cl}]^{+}$ 528.1486, found 528.1489.

\section{9-O-[I-(2-bromobenzyl)-4-ethyl-IH-I, 2, 3-triazole]} berberine chloride (compound II)

Yellow solid, yield 54\%; ${ }^{1} \mathrm{H}$ NMR (300 MHz, DMSO): $\delta 9.66$ (s, 1H), 8.93 (s, 1H), $8.34(\mathrm{~s}, 1 \mathrm{H}), 8.21(\mathrm{~d}, \mathrm{~J}=9.2 \mathrm{~Hz}, 1 \mathrm{H})$, $8.01(\mathrm{~d}, \mathrm{~J}=9.1 \mathrm{~Hz}, 1 \mathrm{H}), 7.79(\mathrm{~s}, 1 \mathrm{H}), 7.60$ (d, J=2.1 Hz, 1H), $7.45-7.38(\mathrm{~m}, 1 \mathrm{H}), 7.37(\mathrm{~d}, \mathrm{~J}=2.1 \mathrm{~Hz}, 1 \mathrm{H}), 7.15-6.97$ (m, 2H), $6.18(\mathrm{~s}, 2 \mathrm{H}), 5.65(\mathrm{~s}, 2 \mathrm{H}), 5.48(\mathrm{~s}, 2 \mathrm{H}), 4.83$ (s, 2H), 4.08 (s, 3H), 3.22-3.02 (m, 2H); ${ }^{13} \mathrm{C} \mathrm{NMR}$ (75 MHz, DMSO): $\delta 151.46,150.40,148.24,146.09,142.88,142.01$, $138.11,134.49,134.15,133.37,132.76,132.23,131.08$, $129.57,128.28,127.11,126.34,124.60,122.49,120.82$, $120.79,108.95,106.16,102.78,66.91,57.54,55.97,50.56$, 27.05; HR-ESI-MS calculated for $\mathrm{C}_{29} \mathrm{H}_{24} \mathrm{BrN}_{4} \mathrm{O}_{4}^{+}[\mathrm{M}+1-\mathrm{Cl}]^{+}$ 573.0980, found 573.0986.

\section{9-O-[I-(3-bromobenzyl)-4-ethyl-IH-I, 2, 3-triazole]}

berberine chloride (compound 12)

Yellow solid, yield 50\%; ${ }^{1} \mathrm{H}$ NMR (300 MHz, DMSO): $\delta 9.67$ $(\mathrm{s}, 1 \mathrm{H}), 8.91(\mathrm{~d}, \mathrm{~J}=7.6 \mathrm{~Hz}, 1 \mathrm{H}), 8.49(\mathrm{~s}, 1 \mathrm{H}), 8.17(\mathrm{~d}, \mathrm{~J}=9.2 \mathrm{~Hz}$, 1H), $7.99(\mathrm{~d}, \mathrm{~J}=9.1 \mathrm{~Hz}, 1 \mathrm{H}), 7.75(\mathrm{~s}, 1 \mathrm{H}), 7.49-7.35(\mathrm{~m}, 2 \mathrm{H})$, $7.24(\mathrm{t}, \mathrm{J}=7.7 \mathrm{~Hz}, 1 \mathrm{H}), 7.19-7.09(\mathrm{~m}, 1 \mathrm{H}), 7.06(\mathrm{~s}, 1 \mathrm{H})$, $6.16(\mathrm{~s}, 2 \mathrm{H}), 5.60(\mathrm{~s}, 2 \mathrm{H}), 5.46(\mathrm{~s}, 2 \mathrm{H}), 4.84(\mathrm{t}, \mathrm{J}=5.8 \mathrm{~Hz}$, $2 \mathrm{H}), 4.07(\mathrm{~d}, \mathrm{~J}=8.0 \mathrm{~Hz}, 3 \mathrm{H}), 3.12(\mathrm{t}, \mathrm{J}=5.8 \mathrm{~Hz}, 2 \mathrm{H}) ;{ }^{13} \mathrm{C} \mathrm{NMR}$ (75 MHz, DMSO): $\delta 151.38,150.33,148.17,145.63,143.16$, $141.82,139.06,137.80,133.31,131.43,131.36,131.05(2 \mathrm{C})$, $127.30,126.92,126.11,124.51,122.39,122.27,120.82$, $120.77,108.91,105.90,102.59,66.83,57.51,55.80,52.39$, 26.83; HR-ESI-MS calculated for $\mathrm{C}_{29} \mathrm{H}_{24} \mathrm{BrN}_{4} \mathrm{O}_{4}^{+}[\mathrm{M}+1-\mathrm{Cl}]^{+}$ 573.0980 , found 573.0986 .

\section{9-O-[I-(4-bromobenzyl)-4-ethyl-I H-I, 2, 3-triazole]} berberine chloride (compound I3)

Yellow solid, yield 58\%; ${ }^{1} \mathrm{H}$ NMR (300 MHz, DMSO) $\delta 9.66$ (s, 1H), $8.93(\mathrm{~s}, 1 \mathrm{H}), 8.55-8.29(\mathrm{~m}, 1 \mathrm{H}), 8.22(\mathrm{~d}, \mathrm{~J}=9.2 \mathrm{~Hz}$, 1H), $8.01(\mathrm{~d}, \mathrm{~J}=8.8 \mathrm{~Hz}, 1 \mathrm{H}), 7.79$ (s, 1H), 7.49 (d, J=7.8 Hz, 2H), 7.08 (s, 3H), $6.18(\mathrm{~s}, 2 \mathrm{H}), 5.52(\mathrm{~d}, \mathrm{~J}=29.6 \mathrm{~Hz}, 2 \mathrm{H})$, $5.47(\mathrm{~s}, 2 \mathrm{H}), 4.82(\mathrm{~s}, 2 \mathrm{H}), 4.07$ (d, J=4.6 Hz, 3H), 3.12 (s, 2H); ${ }^{13} \mathrm{C}$ NMR (75 MHz, DMSO): $\delta$ 151.73, 150.72, $148.55,146.05,143.40,142.18,138.18,136.11,133.63$, 
$132.18,131.56,131.38(2 \mathrm{C}), 130.38,126.86,126.33,124.96$, $122.78,122.26,121.13,120.96,109.41,106.16,102.91$, $67.13,57.77,56.30,52.78,27.06$; HR-ESI-MS calculated for $\mathrm{C}_{29} \mathrm{H}_{24} \mathrm{BrN}_{4} \mathrm{O}_{4}^{+}[\mathrm{M}+1-\mathrm{Cl}]^{+}$573.0980, found 573.0987.

\section{9-O-[I-(2-methylbenzyl)-4-ethyl-I H-I, 2, 3-triazole]} berberine chloride (compound 14)

Yellow solid, yield 61\%; ${ }^{1} \mathrm{H}$ NMR (300 MHz, DMSO): $\delta 9.67$ (s, 1H), 8.92 (s, 1H), 8.42 (s, 1H), 8.20 (d, J=9.2 Hz, 1H), $8.00(\mathrm{~d}, \mathrm{~J}=9.1 \mathrm{~Hz}, 1 \mathrm{H}), 7.78(\mathrm{~s}, 1 \mathrm{H}), 7.36-7.22(\mathrm{~m}, 3 \mathrm{H})$, 7.15-7.05 (m, 2H), $6.18(\mathrm{~s}, 2 \mathrm{H}), 5.60(\mathrm{~s}, 2 \mathrm{H}), 5.47(\mathrm{~s}, 2 \mathrm{H})$, 4.83 (d, J=6.0 Hz, 2H), 4.07 (s, 3H), 3.17-3.07 (m, 2H), 1.30-1.20 (m, 3H); ${ }^{13} \mathrm{C}$ NMR (75 MHz, DMSO): $\delta 150.97$, $149.94,147.83,145.38,145.25,142.68,141.44,138.43$, $137.46,133.35,132.89,130.60,128.04,127.65,126.61$, $126.39,125.64,124.09,122.02,120.43,120.32,108.51$, $105.50,102.22,66.44,57.02,55.35,52.06,29.09,26.39$; HR-ESI-MS calculated for $\mathrm{C}_{30} \mathrm{H}_{27} \mathrm{~N}_{4} \mathrm{O}_{4}^{+}[\mathrm{M}+1-\mathrm{Cl}]^{+} 539.1726$, found 539.1732 .

\section{9-O-[I-(4-methylbenzyl)-4-ethyl-I H-I, 2, 3-triazole]} berberine chloride (compound I5)

Yellow solid, yield 74\%; ${ }^{1} \mathrm{H}$ NMR (300 MHz, DMSO) $\delta 9.62(\mathrm{~s}, 1 \mathrm{H}), 8.94(\mathrm{~d}, \mathrm{~J}=7.7 \mathrm{~Hz}, 1 \mathrm{H}), 8.35(\mathrm{~s}, 1 \mathrm{H})$, 8.26-8.16 (m, 1H), $8.03(\mathrm{t}, \mathrm{J}=11.6 \mathrm{~Hz}, 1 \mathrm{H}), 7.80(\mathrm{~s}, 1 \mathrm{H})$, $7.11-6.91(\mathrm{~m}, 5 \mathrm{H}), 6.18(\mathrm{~s}, 2 \mathrm{H}), 5.48(\mathrm{~d}, \mathrm{~J}=9.8 \mathrm{~Hz}, 4 \mathrm{H})$, $4.76(\mathrm{t}, \mathrm{J}=6.0 \mathrm{~Hz}, 2 \mathrm{H}), 4.08(\mathrm{~s}, 3 \mathrm{H}), 3.08(\mathrm{~d}, \mathrm{~J}=5.9 \mathrm{~Hz}$, 2H), 2.15 (s, 3H); ${ }^{13} \mathrm{C}$ NMR (75 MHz, DMSO) $\delta$ 151.56, $150.37,148.22,145.62,142.96,141.70,137.84,133.50$, $133.30,131.09,129.64,127.92,127.04,125.86,124.51$, $122.56,120.86,120.77,108.93,105.94,102.61,100.02,66.69$, 57.53, 55.71, 52.96, 26.76, 21.00; HR-ESI-MS calculated for $\mathrm{C}_{30} \mathrm{H}_{27} \mathrm{~N}_{4} \mathrm{O}_{4}^{+}[\mathrm{M}+1-\mathrm{Cl}]^{+}$539.1726, found 539.1731.

\section{9-O-[I-(4-tert-butylbenzy I)-4-ethyl-I H-I, 2,}

\section{3-triazole] berberine chloride (compound I6)}

Yellow solid, yield 68\%; ${ }^{1} \mathrm{H}$ NMR (300 MHz, DMSO): $\delta 9.71(\mathrm{~s}, 1 \mathrm{H}), 8.96(\mathrm{~s}, 1 \mathrm{H}), 8.40(\mathrm{~s}, 1 \mathrm{H}), 8.21$ $(\mathrm{d}, \mathrm{J}=9.1 \mathrm{~Hz}, 1 \mathrm{H}), 8.01(\mathrm{~d}, \mathrm{~J}=9.1 \mathrm{~Hz}, 1 \mathrm{H}), 7.80(\mathrm{~s}, 1 \mathrm{H})$, 7.28 (d, J=8.2 Hz, 2H), 7.13-6.96 (m, 3H), 6.17 (s, 2H), $5.54(\mathrm{~s}, 2 \mathrm{H}), 5.46(\mathrm{~s}, 2 \mathrm{H}), 4.88(\mathrm{~s}, 2 \mathrm{H}), 4.06(\mathrm{~s}, 3 \mathrm{H}), 3.15$ (s, 2H), $1.20(\mathrm{~s}, 9 \mathrm{H}) ;{ }^{13} \mathrm{C} \mathrm{NMR}(75 \mathrm{MHz}, \mathrm{DMSO}): \delta 155.73$, $151.21,150.25,145.67,148.07,142.78,141.57,138.13$, $133.60,132.96,130.98,127.28,126.64,125.38,124.41$, $122.13,120.38,120.30,120.22,119.12,108.34,105.65$, $102.35,100.43,78.61,57.21,55.56,52.50,34.45,30.95$, 29.23; HR-ESI-MS calculated for $\mathrm{C}_{33} \mathrm{H}_{33} \mathrm{~N}_{4} \mathrm{O}_{4}^{+}[\mathrm{M}+1-\mathrm{Cl}]^{+}$ 550.2501 , found 550.2507 .
9-O-[I-(3-methoxybenzyl)-4-ethyl-IH-I, 2,

3-triazole] berberine chloride (compound 17)

Yellow solid, yield 65\%; ${ }^{1} \mathrm{H}$ NMR (300 MHz, DMSO): $\delta 9.65$ $(\mathrm{s}, 1 \mathrm{H}), 8.91(\mathrm{~s}, 1 \mathrm{H}), 8.41(\mathrm{~s}, 1 \mathrm{H}), 8.19(\mathrm{~d}, \mathrm{~J}=9.2 \mathrm{~Hz}, 1 \mathrm{H})$, $8.00(\mathrm{~d}, \mathrm{~J}=9.1 \mathrm{~Hz}, 1 \mathrm{H}), 7.78(\mathrm{~s}, 1 \mathrm{H}), 7.22-7.12(\mathrm{~m}, 1 \mathrm{H})$, 7.08 (s, 1H), 6.81-6.73 (m, 2H), 6.64 (d, J=7.5 Hz, 1H), $6.17(\mathrm{~s}, 2 \mathrm{H}), 5.53(\mathrm{~s}, 2 \mathrm{H}), 5.46(\mathrm{~s}, 2 \mathrm{H}), 4.81(\mathrm{t}, \mathrm{J}=5.3 \mathrm{~Hz}, 2 \mathrm{H})$, 4.07 (s, 3H), 3.67 (s, 3H), 3.12 (d, J=5.3 Hz, 2H); ${ }^{13} \mathrm{C}$ NMR (75 MHz, DMSO): $\delta 159.35,150.99,149.88,147.72,145.13$, $142.58,141.32,137.43,137.37,132.86,130.61,129.84$, $126.52,125.49,124.03,122.00,120.38,120.29,119.61$, 113.63, 113.20, 108.43, 105.45, 102.12, 66.32, 57.04, 55.29, 55.10, 52.66, 26.33; HR-ESI-MS calculated for $\mathrm{C}_{30} \mathrm{H}_{27} \mathrm{~N}_{4} \mathrm{O}_{5}^{+}$ $[\mathrm{M}+1-\mathrm{Cl}]^{+}$523.1981, found 523.1987.

\section{9-O-[I-(4-methoxybenzyl)-4-ethyl-IH-I, 2,}

3-triazole] berberine chloride (compound 18)

Yellow solid, yield 78\%; ${ }^{1} \mathrm{H}$ NMR (300 MHz, DMSO): $\delta 9.65$ $(\mathrm{s}, 1 \mathrm{H}), 8.94(\mathrm{~s}, 1 \mathrm{H}), 8.48(\mathrm{~s}, 1 \mathrm{H}), 8.17(\mathrm{~d}, \mathrm{~J}=8.6 \mathrm{~Hz}, 1 \mathrm{H}), 8.01$ (d, J=8.3 Hz, 1H), $7.76(\mathrm{~s}, 1 \mathrm{H}), 7.09$ (d, J=8.1 Hz, 3H), 6.79 $(\mathrm{d}, \mathrm{J}=7.0 \mathrm{~Hz}, 2 \mathrm{H}), 6.16(\mathrm{~s}, 2 \mathrm{H}), 5.47(\mathrm{~s}, 2 \mathrm{H}), 5.44(\mathrm{~s}, 2 \mathrm{H})$, $4.83(\mathrm{~s}, 2 \mathrm{H}), 4.05$ (s, 3H), 3.64 (s, 3H), $3.09(\mathrm{~s}, 2 \mathrm{H}) ;{ }^{13} \mathrm{C} \mathrm{NMR}$ (75 MHz, DMSO): $\delta 159.57,151.57,150.39,148.23,145.68$, $143.03,141.83,137.86,137.34,133.42,131.12,129.75$, $128.45,127.04,125.92,124.63,122.56,120.84,120.56$, 119.12, 114.53, 108.95, 106.04, 102.65, 66.86, 57.62, 55.84, 55.64, 52.80, 26.88; HR-ESI-MS calculated for $\mathrm{C}_{30} \mathrm{H}_{27} \mathrm{~N}_{4} \mathrm{O}_{5}^{+}$ $[\mathrm{M}+1-\mathrm{Cl}]^{+}$523.1981, found 523.1989.

9-O-[4-(trifluoromethoxy)benzyl)-4-ethyl- IH-I, 2, 3-triazole] berberine chloride (compound 19)

Yellow solid, yield 85\%; ${ }^{1} \mathrm{H}$ NMR (300 MHz, DMSO): $\delta 9.67$ $(\mathrm{s}, 1 \mathrm{H}), 8.91(\mathrm{~s}, 1 \mathrm{H}), 8.54(\mathrm{~s}, 1 \mathrm{H}), 8.16(\mathrm{~d}, \mathrm{~J}=9.3 \mathrm{~Hz}, 1 \mathrm{H})$, $8.02(\mathrm{~d}, \mathrm{~J}=9.2 \mathrm{~Hz}, 1 \mathrm{H}), 7.75(\mathrm{~s}, 1 \mathrm{H}), 7.33-7.21(\mathrm{~m}, 4 \mathrm{H})$, $7.05(\mathrm{~s}, 1 \mathrm{H}), 6.14(\mathrm{~s}, 2 \mathrm{H}), 5.62(\mathrm{~s}, 2 \mathrm{H}), 5.45(\mathrm{~s}, 2 \mathrm{H}), 4.84$ (t, J=5.9 Hz, 2H), 4.04 (s, 3H), 3.16-3.06 (m, 2H); ${ }^{13} \mathrm{C} \mathrm{NMR}$ (75 MHz, DMSO): $\delta 151.15,150.02,148.15,147.86$, $145.31,142.79,141.41,137.50,135.58,133.03,130.76$, $129.93,126.62,125.94,124.31,122.12,121.38,120.48$, $120.42,108.59,105.62,102.26,66.37,57.22,55.51,52.01$, 26.50; HR-ESI-MS calculated for $\mathrm{C}_{30} \mathrm{H}_{24} \mathrm{~F}_{3} \mathrm{~N}_{4} \mathrm{O}_{5}^{+}[\mathrm{M}+1-\mathrm{Cl}]^{+}$ 577.1698 , found 577.1702 .

\section{9-O-[I-(3-(trifluoromethyl)benzyl-4-ethyl-I H-I, 2,}

3-triazole] berberine chloride (compound 20)

Yellow solid, yield 83\%; 1H NMR (300 MHz, DMSO): $\delta 9.73$ (s, 1H), 8.95(s, 1H), 8.57(s, 1H), $8.21(\mathrm{~d}, \mathrm{~J}=9.2 \mathrm{~Hz}, 1 \mathrm{H})$, 8.01 (d, J=9.1 Hz, 1H), 7.80 (s, 1H), 7.74(d, J=8.1 Hz, 2H), 
7.40 (d, J=8.0 Hz, 2H), 7.12 (s, 1H), 6.24(s, 2H), 5.77 (s, 2H), $5.52(\mathrm{~s}, 2 \mathrm{H}), 4.93(\mathrm{t}, \mathrm{J}=5.5 \mathrm{~Hz}, 2 \mathrm{H}), 4.14(\mathrm{~s}, 3 \mathrm{H})$, 3.16 (d, J=5.6 Hz, 2H); ${ }^{13} \mathrm{C}$ NMR (75 MHz, DMSO): $\delta 150.80,149.72,147.56,145.04,142.62,141.41,137.22$, $132.67,131.79,130.52,129.76$ (2C), 126.33, 125.36, $124.99,124.88,124.69,124.59,123.84,121.80,120.32$, $120.23,108.37,105.38,102.05,65.78,56.78,54.83,51.50$, 25.54; HR-ESI-MS calculated for $\mathrm{C}_{30} \mathrm{H}_{24} \mathrm{~F}_{3} \mathrm{~N}_{4} \mathrm{O}_{4}^{+}[\mathrm{M}+1-\mathrm{Cl}]^{+}$ 562.1749 , found 562.1756 .

\section{9-O-[I-(4-(trifluoromethyl)benzyl-4-ethyl-I H-I, 2,} 3-triazole] berberine chloride (compound $2 \mathrm{I}$ )

Yellow solid, yield 88\%; ${ }^{1} \mathrm{H}$ NMR (300 MHz, DMSO): $\delta 9.75$ (s, 1H), $8.96(\mathrm{~s}, 1 \mathrm{H}), 8.56(\mathrm{~s}, 1 \mathrm{H}), 8.23(\mathrm{~d}, \mathrm{~J}=9.2 \mathrm{~Hz}, 1 \mathrm{H})$, $8.04(\mathrm{~d}, \mathrm{~J}=9.1 \mathrm{~Hz}, 1 \mathrm{H}), 7.80(\mathrm{~s}, 1 \mathrm{H}), 7.71(\mathrm{~d}, \mathrm{~J}=8.1 \mathrm{~Hz}, 2 \mathrm{H})$, 7.39 (d, J=8.0 Hz, 2H), 7.10 (s, 1H), 6.20 (s, 2H), 5.77 (s, 2H), $5.51(\mathrm{~s}, 2 \mathrm{H}), 4.91$ (t, J=5.5 Hz, 2H), 4.11 (s, 3H), $3.18(\mathrm{~d}, \mathrm{~J}=5.6 \mathrm{~Hz}, 2 \mathrm{H}) ;{ }^{13} \mathrm{C}$ NMR (75 MHz, DMSO): $\delta 150.86,149.68,148.06,145.24,142.66,141.71,137.32$, $132.77,132.09$, 130.32, 129.72, 126.37, 125.34, 124.97, $124.78,124.64,124.53,123.90,121.83,120.40,120.33$, 108.39, 105.41, 102.15, 65.88, 56.48, 54.63, 52.21, 25.04; HR-ESI-MS calculated for $\mathrm{C}_{30} \mathrm{H}_{24} \mathrm{~F}_{3} \mathrm{~N}_{4} \mathrm{O}_{4}^{+}[\mathrm{M}+1-\mathrm{Cl}]^{+}$ 562.1749, found 562.1755.

\section{9-O-[I-(2-benzonitrile)-4-ethyl-IH-I, 2, 3-triazole]} berberine chloride (compound 22)

Yellow solid, yield 79\%; ${ }^{1} \mathrm{H}$ NMR (300 MHz, DMSO): $\delta 9.67$ $(\mathrm{s}, 1 \mathrm{H}), 8.92(\mathrm{~s}, 1 \mathrm{H}), 8.39(\mathrm{~s}, 1 \mathrm{H}), 8.20(\mathrm{~d}, \mathrm{~J}=9.1 \mathrm{~Hz}, 1 \mathrm{H})$, 8.00 (d, J=9.3 Hz, 1H), 7.90-7.75 (m, 2H), 7.65 (t, J=7.4 $\mathrm{Hz}, 1 \mathrm{H}), 7.49$ (t, J=7.5 Hz, 1H), 7.23 (d, J=7.3 Hz, 1H), 7.08 (s, 1H), $6.18(\mathrm{~s}, 2 \mathrm{H}), 5.78(\mathrm{~s}, 2 \mathrm{H}), 5.49(\mathrm{~s}, 2 \mathrm{H}), 4.84(\mathrm{~s}, 2 \mathrm{H})$, 4.07 (s, 3H), 3.14 (s, 2H); ${ }^{13} \mathrm{C}$ NMR (75 MHz, DMSO): $\delta 151.14,150.00,147.63,145.14,142.65,141.36,141.29$, $137.36,132.88,132.78,130.65,128.32,126.48,126.07$, $124.14,122.06,120.32,118.36,110.78,108.37,105.38$, $102.17,66.20,57.12,55.29,52.12,26.31$; HR-ESI-MS calculated for $\mathrm{C}_{30} \mathrm{H}_{24} \mathrm{~N}_{5} \mathrm{O}_{4}^{+}[\mathrm{M}+1-\mathrm{Cl}]^{+} 519.1828$, found 519.1832 .

\section{9-O-[I-(4-benzonitrile)-4-ethyl- I H-I, 2, 3-triazole]} berberine chloride (compound 23)

Yellow solid, yield 83\%; ${ }^{1} \mathrm{H}$ NMR (300 MHz, DMSO): $\delta 9.68$ (s, 1H), $8.92(\mathrm{~s}, 1 \mathrm{H}), 8.52(\mathrm{~s}, 1 \mathrm{H}), 8.20(\mathrm{~d}, \mathrm{~J}=9.0 \mathrm{~Hz}, 1 \mathrm{H}), 8.01$ (d, J=8.9 Hz, 1H), 7.82-7.66 (m, 3H), $7.23(\mathrm{~d}, \mathrm{~J}=7.7 \mathrm{~Hz}, 2 \mathrm{H})$, $7.05(\mathrm{~s}, 1 \mathrm{H}), 6.16(\mathrm{~s}, 2 \mathrm{H}), 5.71(\mathrm{~s}, 2 \mathrm{H}), 5.48(\mathrm{~s}, 2 \mathrm{H})$, 4.82 (s, 2H), 4.08 (s, 3H), 3.07 (s, 2H); ${ }^{13} \mathrm{C}$ NMR (75 MHz, DMSO): $\delta 151.04,150.00,147.83,145.18,142.68,141.46$,
$141.24,137.35,132.85,132.61,130.56,128.28,126.52$, $126.01,124.11,122.02,120.30,118.38,110.85,108.41$, 105.41, 102.14, 66.23, 57.09, 55.31, 52.09, 26.33; HR-ESI-MS calculated for $\mathrm{C}_{30} \mathrm{H}_{24} \mathrm{~N}_{5} \mathrm{O}_{4}^{+}[\mathrm{M}+1-\mathrm{Cl}]^{+} 519.1828$, found 519.1833 .

\section{9-O-[I-(4-nitrobenzyl)-4-ethyl- I H-I, 2, 3-triazole]} berberine chloride (compound 24)

Yellow solid, yield 85\%; ${ }^{1} \mathrm{H}$ NMR (300 MHz, DMSO): $\delta 9.70$ (s, 1H), 8.91 (s, 1H), $8.63(\mathrm{~s}, 1 \mathrm{H}), 8.24-8.04(\mathrm{~m}, 3 \mathrm{H}), 7.99$ (d, J=9.1 Hz, 1H), 7.72 (s, 1H), 7.36 (d, J=8.7 Hz, 2H), 7.03 (s, 1H), $6.16(\mathrm{~s}, 2 \mathrm{H}), 5.77$ (d, J=9.0 Hz, 2H), $5.48(\mathrm{~s}, 2 \mathrm{H})$, 4.87 (t, J=5.6 Hz, 2H), 4.08 (d, J=2.9 Hz, 3H), 3.07 (d, $\mathrm{J}=5.4 \mathrm{~Hz}, 2 \mathrm{H}) ;{ }^{13} \mathrm{C}$ NMR (75 MHz, DMSO): $\delta 151.45$, $150.34,148.17,147.51,145.64,143.89,143.19,141.73$, $137.74,133.29,132.45,130.95,129.22,126.95,126.55$, $124.53,124.22,122.42,120.70,118.58,110.65,108.82$, $105.83,102.58,66.75,57.55,55.75,52.27,26.78$; HRESI-MS calculated for $\mathrm{C}_{29} \mathrm{H}_{24} \mathrm{~N}_{5} \mathrm{O}_{6}^{+}[\mathrm{M}+1-\mathrm{Cl}]^{+} 539.1726$, found 539.173 .

9-O-[I-(4-methyl formate benzyl)-4-ethyl-I H-I, 2, 3-triazole] berberine chloride (compound 25)

Yellow solid, yield 69\%; ${ }^{1} \mathrm{H}$ NMR (300 MHz, DMSO): $\delta 9.61$ (s, 1H), $8.87(\mathrm{~s}, 1 \mathrm{H}), 8.41(\mathrm{~s}, 1 \mathrm{H}), 8.21(\mathrm{~d}, \mathrm{~J}=8.5 \mathrm{~Hz}, 1 \mathrm{H}), 8.01$ (d, J=7.9 Hz, 1H), 7.84-7.68 (m, 3H), 7.11 (d, J=7.4 Hz, 2H), 7.03 (s, 1H), 6.17 (s, 2H), 5.65 (s, 2H), 5.49 (s, 2H), 4.75 (s, 2H), 4.08 (s, 3H), 3.81 (s, 3H); ${ }^{13} \mathrm{C}$ NMR (75 MHz, DMSO): $\delta 165.74,151.30,150.06,147.84,145.20,142.71$, $141.24,141.09,137.38,132.83,130.54,129.45,129.20$, $127.52,126.65,125.91,124.16,122.19,120.25,108.43$, 105.48, 102.19, 66.14, 57.06, 55.35, 52.18, 26.36; HR-ESI-MS calculated for $\mathrm{C}_{31} \mathrm{H}_{27} \mathrm{~N}_{4} \mathrm{O}_{6}{ }^{+}[\mathrm{M}+1-\mathrm{Cl}]^{+} 552.1930$, found 552.1936 .

9-O-[I-(4-(methylsulfonyl)benzyl-4-ethyl-I H-I, 2, 3-triazole) berberine chloride (compound 26)

Yellow solid, yield 73\%; ${ }^{1} \mathrm{H}$ NMR (300 MHz, DMSO): $\delta 9.73(\mathrm{~s}, 1 \mathrm{H}), 8.93$ (s, 1H), $8.53(\mathrm{~s}, 1 \mathrm{H}), 8.19$ (d, J=9.2 Hz, $1 \mathrm{H}), 8.01(\mathrm{~d}, \mathrm{~J}=9.2 \mathrm{~Hz}, 1 \mathrm{H}), 7.89(\mathrm{~d}, \mathrm{~J}=8.3 \mathrm{~Hz}, 2 \mathrm{H})$, $7.77(\mathrm{~s}, 1 \mathrm{H}), 7.45$ (d, J=8.3 Hz, 2H), 7.07 (s, 1H), 6.17 (s, 2H), 5.77 (s, 2H), 5.47 (s, 2H), $4.90(\mathrm{t}, \mathrm{J}=5.6 \mathrm{~Hz}, 2 \mathrm{H})$, 4.06 (s, 3H), 3.19 (s, 3H), 3.17 (s, 2H); ${ }^{13} \mathrm{C}$ NMR (75 MHz, DMSO): $\delta 150.86,149.91,147.75,145.27,142.83,141.71$, $141.57,140.52,137.47,132.94,130.65,128.60,127.45$, $126.54,125.84,124.01,121.87,120.36,120.27,108.45$, 105.47, 102.13, 66.54, 57.08, 55.40, 52.12, 43.44, 26.39; HR-ESI-MS calculated for $\mathrm{C}_{30} \mathrm{H}_{27} \mathrm{~N}_{4} \mathrm{O}_{6} \mathrm{~S}^{+}[\mathrm{M}+1-\mathrm{Cl}]^{+}$ 572.1651 , found 572.1656 . 
9-O-[I-(2, 4-difluorobenzyl)-4-ethyl-I H-I, 2 ,

3-triazole] berberine chloride (compound 27)

Yellow solid, yield 74\%; ${ }^{1} \mathrm{H}$ NMR (300 MHz, DMSO): $\delta 9.70$ $(\mathrm{s}, 1 \mathrm{H}), 8.96(\mathrm{~d}, \mathrm{~J}=8.6 \mathrm{~Hz}, 1 \mathrm{H}), 8.46(\mathrm{~s}, 1 \mathrm{H}), 8.23(\mathrm{~d}, \mathrm{~J}=9.2 \mathrm{~Hz}$, 1H), $8.05(\mathrm{t}, \mathrm{J}=7.6 \mathrm{~Hz}, 1 \mathrm{H}), 7.82(\mathrm{~d}, \mathrm{~J}=6.8 \mathrm{~Hz}, 1 \mathrm{H}), 7.37$ (tt, J=13.3, 5.2 Hz, 2H), 7.15-7.00 (m, 2H), $6.20(\mathrm{~s}, 2 \mathrm{H})$, $5.61(\mathrm{~s}, 2 \mathrm{H}), 5.49(\mathrm{~s}, 2 \mathrm{H}), 4.88(\mathrm{t}, \mathrm{J}=5.8 \mathrm{~Hz}, 2 \mathrm{H}), 4.11$ (d, J=6.2 Hz, 3H), 3.16 (t, J=5.8 Hz, 2H); ${ }^{13} \mathrm{C}$ NMR $(75 \mathrm{MHz}$, DMSO): $\delta 150.95,149.91,147.73,145.20,142.70,141.45$, $137.40,132.88,131.89,130.60,126.55,125.52,124.02$, $121.95,120.34,120.29,118.02,117.80,117.35,117.06$, $108.44,105.44,102.13,100.24,66.37,57.06,55.48,51.58$, 26.45; HR-ESI-MS calculated for $\mathrm{C}_{29} \mathrm{H}_{23} \mathrm{~F}_{2} \mathrm{~N}_{4} \mathrm{O}_{4}^{+}[\mathrm{M}+1-\mathrm{Cl}]^{+}$ 530.1687 , found 530.1692 .

\section{9-O-[I-(2, 6-difluorobenzyl)-4-ethyl-I H-I, 2,} 3-triazole] berberine chloride (compound 28)

Yellow solid, yield 63\%; ${ }^{1} \mathrm{H}$ NMR (300 MHz, DMSO): $\delta 9.66$ (s, 1H), 8.93 (s, 1H), 8.34 (s, 1H), 8.19 (d, J=8.9 Hz, 1H), 8.00 (d, J=8.7 Hz, 1H), 7.79 (s, 1H), 7.43 (s, 1H), 7.09 (s, 3H), 6.18 (s, 2H), $5.63(\mathrm{~s}, 2 \mathrm{H}), 5.44(\mathrm{~s}, 2 \mathrm{H}), 4.85(\mathrm{~s}, 2 \mathrm{H}), 4.06(\mathrm{~s}, 3 \mathrm{H})$, 3.15 (s, 2H); ${ }^{13} \mathrm{C}$ NMR (75 MHz, DMSO): $\delta 159.26,150.98$, $150.15,149.83,147.96,145.19,142.77,141.62,137.76$, $133.14,131.69,130.66,126.66,125.65,124.09,122.02$, $120.49,112.05,111.93,111.79,108.53,108.30,105.51$, $102.19,99.15,66.29,57.08,55.47,26.46$; HR-ESI-MS calculated for $\mathrm{C}_{29} \mathrm{H}_{23} \mathrm{~F}_{2} \mathrm{~N}_{4} \mathrm{O}_{4}^{+}[\mathrm{M}+1-\mathrm{Cl}]^{+} 530.1687$, found 530.1691 .

9-O-[I-(3, 4-difluorobenzyl)-4-ethyl- IH-I, 2, 3-triazole] berberine chloride (compound 29)

Yellow solid, yield 67\%; ${ }^{1} \mathrm{H}$ NMR (300 MHz, DMSO): $\delta 9.70$ $(\mathrm{s}, 1 \mathrm{H}), 8.97(\mathrm{~d}, \mathrm{~J}=16.3 \mathrm{~Hz}, 1 \mathrm{H}), 8.45(\mathrm{~s}, 1 \mathrm{H}), 8.25(\mathrm{t}, \mathrm{J}=8.7 \mathrm{~Hz}$, 1H), $8.06(\mathrm{dd}, \mathrm{J}=15.2,9.2 \mathrm{~Hz}, 1 \mathrm{H}), 7.82(\mathrm{~d}, \mathrm{~J}=6.8 \mathrm{~Hz}, 1 \mathrm{H})$, 7.38 (dt, J=17.9, $9.0 \mathrm{~Hz}, 2 \mathrm{H}), 7.10(\mathrm{~d}, \mathrm{~J}=10.8 \mathrm{~Hz}, 2 \mathrm{H})$, $6.21(\mathrm{~s}, 2 \mathrm{H}), 5.61(\mathrm{~s}, 2 \mathrm{H}), 5.49(\mathrm{~s}, 2 \mathrm{H}), 4.88(\mathrm{~s}, 2 \mathrm{H}), 4.10$ (s, 3H), 3.21-3.06 (m, 2H); ${ }^{13} \mathrm{C}$ NMR (75 MHz, DMSO): $\delta$ 150.97, 149.92, 147.75, 145.62, 142.71, 141.39, 137.75, $137.42,133.71,132.89,130.62,126.56,125.52,124.05$, $121.97,120.36,120.28,117.98,117.88,117.30,117.12$, 108.46, 105.45, 102.14, 66.37, 57.07, 55.36, 51.60, 26.36; HR-ESI-MS calculated for $\mathrm{C}_{29} \mathrm{H}_{23} \mathrm{~F}_{2} \mathrm{~N}_{4} \mathrm{O}_{4}{ }^{+}[\mathrm{M}+1-\mathrm{Cl}]^{+}$ 530.1687, found 530.1690 .

9-O-[I-(4-chloro-2-fluorobenzyl)-4-ethyl-I H-I, 2, 3-triazole] berberine chloride (compound 30) Yellow solid, yield 66\%; ${ }^{1} \mathrm{H}$ NMR (300 MHz, DMSO): $\delta 9.64(\mathrm{~s}, 1 \mathrm{H}), 8.91(\mathrm{~s}, 1 \mathrm{H}), 8.24(\mathrm{~d}, \mathrm{~J}=37.4 \mathrm{~Hz}, 2 \mathrm{H})$,
7.90 (d, J=64.3 Hz, 2H), 7.36 (d, J=23.5 Hz, 3H), 7.08 (s, 1H), $6.18(\mathrm{~s}, 2 \mathrm{H}), 5.66(\mathrm{~s}, 2 \mathrm{H}), 5.44(\mathrm{~s}, 2 \mathrm{H}), 4.82(\mathrm{~s}, 2 \mathrm{H})$, 4.06 (s, 3H), 3.14 (s, 2H); ${ }^{13} \mathrm{C}$ NMR (75 MHz, DMSO): $\delta 159.56,151.01,149.92,147.77,145.23,142.20,141.31$, $137.39,134.82,132.89,131.88,131.75,130.62,126.51$, $125.73,124.08,122.02,120.98,120.38,115.06,114.77$, 108.48, 105.45, 102.15, 66.24, 57.03, 55.38, 44.47, 26.37; HR-ESI-MS calculated for $\mathrm{C}_{29} \mathrm{H}_{23} \mathrm{ClFN}_{4} \mathrm{O}_{4}^{+}[\mathrm{M}+1-\mathrm{Cl}]^{+}$ 545.1391, found 545.1397.

\section{9-O-[I-(2-bromo-4-fluorobenzyl)-4-ethyl-I H-I, 2,} 3-triazole] berberine chloride (compound 3I)

Yellow solid, yield 62\%; ${ }^{1} \mathrm{H}$ NMR (300 MHz, DMSO): $\delta 9.66$ (s, 1H), $8.91(\mathrm{~s}, 1 \mathrm{H}), 8.43(\mathrm{~s}, 1 \mathrm{H}), 8.20(\mathrm{~d}, \mathrm{~J}=9.0 \mathrm{~Hz}, 1 \mathrm{H}), 7.99$ (d, J=9.1 Hz, 1H), 7.77 (s, 1H), 7.60 (d, J=8.1 Hz, 1H), 7.21 (d, J=9.5 Hz, 1H), 7.07 (s, 1H), 6.90 (d, J=8.4 Hz, 1H), 6.16 (s, 2H), 5.59 (s, 2H), $5.46(\mathrm{~s}, 2 \mathrm{H}), 4.83(\mathrm{~s}, 2 \mathrm{H}), 4.07$ (s, 3H), 3.10 (s, 2H); ${ }^{13} \mathrm{C}$ NMR (75 MHz, DMSO): $\delta 150.93,149.91$, 147.74, 145.19, 142.69, 141.35, 138.22, 138.18, 137.39, $133.82,132.84,130.57,126.54,125.64,125.33,123.99$, 121.94, 120.32, 120.23, 116.25, 115.95, 108.42, 105.45, 102.10, 66.33, 57.04, 55.29, 51.54, 26.32; HR-ESI-MS calculated for $\mathrm{C}_{29} \mathrm{H}_{23} \mathrm{BrFN}_{4} \mathrm{O}_{4}^{+}[\mathrm{M}+1-\mathrm{Cl}]^{+} 590.0886$, found 590.0890 .

9-O-[I-(2, 4-dichlorobenzyl)-4-ethyl-IH-I, 2, 3-triazole] berberine chloride (compound 32)

Yellow solid, yield 73\%; ${ }^{1} \mathrm{H}$ NMR (300 MHz, DMSO): $\delta 9.67$ (s, 1H), 8.92 (s, 1H), 8.31 (s, 1H), 8.19 (d, J=8.1 Hz, 1H), 8.01 $(\mathrm{s}, 1 \mathrm{H}), 7.79(\mathrm{~s}, 1 \mathrm{H}), 7.60(\mathrm{~s}, 1 \mathrm{H}), 7.22(\mathrm{~s}, 1 \mathrm{H}), 7.03(\mathrm{~d}, \mathrm{~J}=28.1$ $\mathrm{Hz}, 2 \mathrm{H}), 6.18(\mathrm{~s}, 2 \mathrm{H}), 5.64(\mathrm{~s}, 2 \mathrm{H}), 5.49(\mathrm{~s}, 2 \mathrm{H}), 4.83(\mathrm{~s}, 2 \mathrm{H})$, 4.07 (s, 3H), 3.13 (s, 2H); ${ }^{13} \mathrm{C}$ NMR (75 MHz, DMSO): $\delta 151.62,150.58,145.84,143.12,139.79,135.46,133.39$, $131.24,130.96,130.85,128.76,127.14,126.45,123.38$, $122.65,122.34,120.93,120.84,109.05,105.99,102.84$, 102.77, 98.27, 96.17, 67.35, 58.44, 56.21, 54.21, 27.63; HR-ESI-MS calculated for $\mathrm{C}_{29} \mathrm{H}_{23} \mathrm{Cl}_{2} \mathrm{~N}_{4} \mathrm{O}_{4}{ }^{+}[\mathrm{M}+1-\mathrm{Cl}]^{+}$ 562.1096, found 562.1094.

\section{9-O-[I-(2, 5-dichlorobenzyl)-4-ethyl-I H-I, 2,}

\section{3-triazole] berberine chloride (compound 33)}

Yellow solid, yield 53\%; ${ }^{1} \mathrm{H}$ NMR (300 MHz, DMSO): $\delta 9.64$ (s, 1H), $8.91(\mathrm{~s}, 1 \mathrm{H}), 8.26(\mathrm{~s}, 1 \mathrm{H}), 8.18(\mathrm{~d}, \mathrm{~J}=9.2 \mathrm{~Hz}, 1 \mathrm{H})$, 7.99 (d, J=9.1 Hz, 1H), 7.78 (s, 1H), 7.48 (d, J=7.5 Hz, 2H), 7.37 (dd, J=9.0, $7.1 \mathrm{~Hz}, 1 \mathrm{H}), 7.08$ (s, 1H), 6.18 (s, 2H), 5.74 (d, J=5.8 Hz, 2H), 5.44 (s, 2H), 4.81 (s, 2H), 4.06 (s, 3H), 3.13 (s, 2H); ${ }^{13} \mathrm{C}$ NMR (75 MHz, DMSO): $\delta 151.03,149.93$, $147.78,145.24,142.06,141.27,137.38,135.83,132.90$, 
$131.64,130.60,130.26,128.93,126.51,125.69,124.09$, $122.05,120.38,120.33,108.50,105.46,102.16,66.19$, 57.04, 55.39, 48.62, 30.73, 26.37; HR-ESI-MS calculated for $\mathrm{C}_{29} \mathrm{H}_{23} \mathrm{Cl}_{2} \mathrm{~N}_{4} \mathrm{O}_{4}^{+}[\mathrm{M}+1-\mathrm{Cl}]^{+}$562.1096, found 562.1095.

\section{9-O-[I-(2, 6-dichlorobenzyl)-4-ethyl-IH-I, 2,} 3-triazole] berberine chloride (compound 34) Yellow solid, yield 58\%; BBR-57 ${ }^{1} \mathrm{H}$ NMR (300 MHz, DMSO): $\delta 9.65$ (s, 1H), 8.92 (s, 1H), 8.32 (s, 1H), 8.18 (d, J=9.2 Hz, 1H), 7.99 (d, J=9.1 Hz, 1H), 7.78 (s, 1H), 7.41 (td, J=8.2, 6.2 Hz, 1H), 7.35-7.20 (m, 2H), 7.08 (s, 1H), 6.17 $(\mathrm{s}, 2 \mathrm{H}), 5.66(\mathrm{~s}, 2 \mathrm{H}), 5.44(\mathrm{~s}, 2 \mathrm{H}), 4.83(\mathrm{t}, \mathrm{J}=5.8 \mathrm{~Hz}, 2 \mathrm{H})$, 4.06 (s, 3H), 3.15 (d, J=5.9 Hz, 2H); ${ }^{13} \mathrm{C}$ NMR (75 MHz, DMSO): $\delta 162.85,159.53,150.97,149.87,147.72,145.20$, $142.18,141.30,137.34,134.79,132.86,131.85,130.57$, $126.47,125.74,124.04,121.98,120.95,120.34,115.03$, 114.74, 108.44, 105.42, 102.12, 66.23, 57.00, 55.34, 44.42, 26.34; HR-ESI-MS calculated for $\mathrm{C}_{29} \mathrm{H}_{23} \mathrm{Cl}_{2} \mathrm{~N}_{4} \mathrm{O}_{4}^{+}[\mathrm{M}+1-\mathrm{Cl}]^{+}$ 562.1096, found 562.1092.

\section{9-O-[I-(3, 4-dichlorobenzyl)-4-ethyl-IH-I, 2,}

\section{3-triazole] berberine chloride (compound 35)}

Yellow solid, yield 76\%; ${ }^{1} \mathrm{H}$ NMR (300 MHz, DMSO): $\delta 9.66$ (s, 1H), $8.88(\mathrm{~s}, 1 \mathrm{H}), 8.47$ (s, 1H), 8.18 (d, J=9.2 Hz, 1H), 8.00 (d, J=9.1 Hz, 1H), 7.75 (s, 1H), 7.52 (dd, J=10.4, 4.9 Hz, 2H), 7.17-7.03 (m, 2H), 6.16 (s, 2H), 5.60 (s, 2H), $5.46(\mathrm{~s}, 2 \mathrm{H})$, $4.83(\mathrm{~d}, \mathrm{~J}=5.3 \mathrm{~Hz}, 2 \mathrm{H}), 4.07(\mathrm{~s}, 3 \mathrm{H}), 3.12$ (d, J=5.5 Hz, $2 \mathrm{H}) ;{ }^{13} \mathrm{C}$ NMR (75 MHz, DMSO): $\delta$ 150.92, 149.89, $147.74,145.15,142.62,141.30,137.32,136.81,132.85$, $131.23,131.00,130.93,130.57,129.88,128.09,126.45$, $125.57,123.90,121.92,120.37,120.28,108.46,105.22$, 101.88, 66.19, 56.81, 55.30, 51.21, 26.16; HR-ESI-MS calculated for $\mathrm{C}_{29} \mathrm{H}_{23} \mathrm{Cl}_{2} \mathrm{~N}_{4} \mathrm{O}_{4}{ }^{+}[\mathrm{M}+1-\mathrm{Cl}]^{+} 562.1096$, found 562.1094 .

\section{9-O-[I-(2, 4, 6-trichlorobenzyl)-4-ethyl-IH-I, 2,}

\section{3 -triazole] berberine chloride (compound 36)}

Yellow solid, yield 63\%; ${ }^{1} \mathrm{H}$ NMR (300 MHz, DMSO): $\delta 9.57$ (s, 1H), 8.90 (s, 1H), 8.17 (d, J=9.1 Hz, 1H), 8.07-7.92 $(\mathrm{m}, 2 \mathrm{H}), 7.81(\mathrm{~s}, 1 \mathrm{H}), 6.75(\mathrm{~s}, 2 \mathrm{H}), 6.18(\mathrm{~s}, 2 \mathrm{H}), 5.44(\mathrm{~s}, 4 \mathrm{H})$, 4.65 (s, 2H), 4.05 (s, 3H), $3.10(\mathrm{~s}, 2 \mathrm{H}) ;{ }^{13} \mathrm{C} \mathrm{NMR}(75 \mathrm{MHz}$, DMSO): $\delta 151.15,149.93,147.80,145.19,141.93,141.05$, $137.72,137.53,137.32,132.81,131.02,130.98,130.59$, $128.98,128.62,126.51,124.99,124.06,122.19,120.40$, 120.25, 108.50, 105.50, 102.29, 66.02, 57.03, 55.17, 47.50, 19.22; HR-ESI-MS calculated for $\mathrm{C}_{29} \mathrm{H}_{22} \mathrm{Cl}_{3} \mathrm{~N}_{4} \mathrm{O}_{4}^{+}[\mathrm{M}+1-\mathrm{Cl}]^{+}$ 596.0706, found 596.0708.

\section{Cell culture}

Human umbilical vein endothelial cell (HUVEC), SW-1990 (human pancreatic carcinoma), and SMMC-7721 (human liver carcinoma) cell lines were provided by Research and Development Center for New Drugs, School of Pharmacy, Second Military Medical University, Shanghai, People's Republic of China. MCF-7 (human breast adenocarcinoma) cells were provided by the Shanghai Institute of Materia Medica (Chinese Academy of Sciences). All adherent cell lines, including MCF-7, SW-1990, and SMMC-7721, were cultured in a humidified $5 \% \mathrm{CO}_{2}$ atmosphere at $37^{\circ} \mathrm{C}$. Cells were cultured in Dulbecco's Modified Eagle's Medium supplemented with $10 \%$ fetal bovine serum and 100 units $/ \mathrm{mL}$ penicillin; $0.1 \mathrm{mg} / \mathrm{mL}$ of streptomycin and HUVEC were cultivated in Endothelial Cell Growth Medium. This medium was prepared with $485 \mathrm{~mL}$ of Endothelial Basal Medium (without serum) and $15 \mathrm{~mL}$ of growth supplement (3\%) of fetal bovine serum. HUVEC cells were cultivated between passages 1 and 7 .

\section{Cytotoxic activity}

The in vitro cytotoxicity of the newly synthesized target compounds 4-36 against MCF-7, SW-1990, SMMC-7721, and HUVEC cell lines was measured by an assay was based on the cleavage of the yellow tetrazolium salt MTT (3-(4,5-Dimethylthiazol-2-yl)-2,5-diphenyltetrazolium bromide) (Sigma-Aldrich) to form purple formazan crystals in viable cells. The cells were plated in 96-well culture plates at a density of 10,000 (MCF-7), 5,000 (SW-1990), 6,000 (SMMC-7721), and 5,000 (HUVEC) cells per well and incubated for 24 hours at $37^{\circ} \mathrm{C}$ in a $5 \% \mathrm{CO}_{2}$ incubator. The compounds were dissolved in DMSO and diluted with culture medium. The compounds were added to the wells at final concentrations of $1.95,3.9,7.8,15.625,31.25,62.5$, 125 , and $250 \mu \mathrm{M}$ before incubation at $37^{\circ} \mathrm{C}$ in a $5 \% \mathrm{CO}_{2}$ incubator for 48 hours. After that, the cells were treated with $10 \%$ volume ratio (v/v) MTT dye solution $(5 \mathrm{mg} / \mathrm{mL})$ for 4 hours. The media with MTT solution was replaced with DMSO $(150 \mu \mathrm{L})$. The 96-well culture plates were then gently shaken in the dark for 30 minutes, and absorbance at $570 \mathrm{~nm}$ and $630 \mathrm{~nm}$ (background) was measured with a microtiter plate reader. The half-maximal inhibitory concentration $\left(\mathrm{IC}_{50}\right)$ value was determined from the chart of cell viability $(\%)$ against compound dose $(\mu \mathrm{M}){ }^{34}$

In vitro cytotoxic activities were evaluated for all synthesized compounds against the MCF-7, SW-1990, SMMC-7721, and HUVEC cell lines. Berberine was taken 
as the reference standard in this study. Berberine and compounds 4-36 were dissolved in DMSO and diluted with culture medium containing $0.1 \%$ DMSO. Cells were treated with culture medium containing $0.1 \%$ DMSO. The percent inhibition of viability at each concentration of the compounds was calculated with respect to the control, and $\mathrm{IC}_{50}$ values were estimated with the software SPSS version 16.0 for Windows (SPSS Inc., Chicago, IL, USA). Each experiment was repeated three times, and the results are summarized in Table 1. The response parameter calculated was $\mathrm{IC}_{50}$ values (Table 1), which corresponded to the compound concentration causing $50 \%$ mortality in net cells.

\section{Statistical analysis}

Each experiment was performed at least in triplicate. The data are presented as mean \pm standard deviation. Using analysis of variance, statistical significance was determined. Mean values with probability values of $P<0.05$ were taken as statistically significant.

Table I In vitro cytotoxicity $\left(\mathrm{IC}_{50}, \mu \mathrm{M}\right)^{\mathrm{a}}$ of the title compounds 4-36 against three human cancer cell lines and the human umbilical vein endothelial cell line

\begin{tabular}{|c|c|c|c|c|c|}
\hline \multirow[t]{2}{*}{ Compound } & \multirow[t]{2}{*}{$\mathbf{R}$} & \multicolumn{4}{|l|}{$I C_{50}(\mu M)$} \\
\hline & & MCF-7 & SW-1990 & SMMC-772I & HUVEC \\
\hline 4 & $\mathrm{H}$ & $77.87 \pm 10.26$ & $28.58 \pm 3.77$ & $35.39 \pm 4.02$ & $71.92 \pm 9.04$ \\
\hline 5 & $2-\mathrm{F}$ & $75.01 \pm 9.35$ & $64.03 \pm 8.46$ & $44.52 \pm 4.88$ & $44.00 \pm 5.03$ \\
\hline 6 & $3-\mathrm{F}$ & $69.88 \pm 8.73$ & $62.59 \pm 7.85$ & $51.49 \pm 6.03$ & $44.26 \pm 4.50$ \\
\hline 7 & $4-\mathrm{F}$ & $73.54 \pm|0.2|$ & $53.63 \pm 6.33$ & $54.58 \pm 5.98$ & $49.82 \pm 6.13$ \\
\hline 8 & $2-\mathrm{Cl}$ & $79.70 \pm I 1.43$ & $40.66 \pm 4.26$ & $39.97 \pm 4.07$ & $33.15 \pm 3.82$ \\
\hline 9 & $3-\mathrm{Cl}$ & $66.09 \pm 7.12$ & $46.80 \pm 4.26$ & $41.10 \pm 3.69$ & $48.17 \pm 4.90$ \\
\hline 10 & $4-\mathrm{Cl}$ & $45.90 \pm 4.46$ & $37.93 \pm 3.52$ & $43.67 \pm 4.91$ & $31.45 \pm 3.22$ \\
\hline II & $2-\mathrm{Br}$ & $25.50 \pm 3.40$ & $25.21 \pm 3.12$ & $24.31 \pm 3.69$ & $13.9 \mid \pm 2.46$ \\
\hline 12 & $3-\mathrm{Br}$ & $66.42 \pm 7.26$ & $50.45 \pm 6.84$ & $33.49 \pm 5.01$ & $25.84 \pm 4.73$ \\
\hline 13 & $4-B r$ & $47.10 \pm 6.93$ & $30.39 \pm 4.89$ & $20.02 \pm 2.37$ & $11.16 \pm 2.60$ \\
\hline 14 & $2-\mathrm{CH}_{3}$ & $40.76 \pm 5.92$ & $23.69 \pm 3.47$ & $19.95 \pm 2.33$ & $67.69 \pm 8.95$ \\
\hline 15 & $4-\mathrm{CH}_{3}$ & $37.83 \pm 4.66$ & $32.03 \pm 4.23$ & $39.37 \pm 4.98$ & $|45.7| \pm 19.01$ \\
\hline 16 & $4-\mathrm{C}\left(\mathrm{CH}_{3}\right)_{3}$ & $15.80 \pm 2.14$ & $8.54 \pm 1.97$ & $11.87 \pm 1.83$ & $25.49 \pm 3.24$ \\
\hline 17 & $3-\mathrm{OCH}_{3}$ & $41.24 \pm 6.90$ & $32.64 \pm 5.67$ & $21.67 \pm 3.35$ & $61.66 \pm 6.78$ \\
\hline 18 & $4-\mathrm{OCH}_{3}$ & $46.67 \pm 5.82$ & $35.64 \pm 4.21$ & $|7.36 \pm 2.3|$ & $71.18 \pm 9.03$ \\
\hline 19 & $4-\mathrm{OCF}_{3}$ & $36.68 \pm 5.63$ & $28.99 \pm 3.26$ & $24.97 \pm 3.41$ & $10.66 \pm 1.45$ \\
\hline 20 & $3-\mathrm{CF}_{3}$ & $86.22 \pm 10.48$ & $39.29 \pm 3.76$ & $32.16 \pm 5.20$ & $42.60 \pm 4.56$ \\
\hline 21 & $4-\mathrm{CF}_{3}$ & $68.07 \pm 7.56$ & $36.80 \pm 3.45$ & $23.28 \pm 2.63$ & $14.40 \pm 2.04$ \\
\hline 22 & $2-\mathrm{CN}$ & $209.35 \pm 21.58$ & $80.99 \pm 10.54$ & $63.99 \pm 8.26$ & $14.77 \pm 2.54$ \\
\hline 23 & $4-\mathrm{CN}$ & $174.37 \pm 20.47$ & $101.07 \pm 15.73$ & $57.31 \pm 6.26$ & $47.28 \pm 5.34$ \\
\hline 24 & $4-\mathrm{NO}_{2}$ & $206.47 \pm 23.59$ & $54.23 \pm 6.55$ & $63.81 \pm 8.90$ & $36.47 \pm 3.46$ \\
\hline 25 & $4-\mathrm{CO}_{2} \mathrm{CH}_{3}$ & $248.55 \pm 20.13$ & $165.27 \pm 18.94$ & $53.49 \pm 5.84$ & $48.89 \pm 5.68$ \\
\hline 26 & $4-\mathrm{SO}_{2} \mathrm{CH}_{3}$ & $206.84 \pm 19.65$ & $100.76 \pm 9.01$ & $69.68 \pm 8.93$ & $74.62 \pm 9.21$ \\
\hline 27 & $2,4-2 F$ & $70.73 \pm 6.45$ & $68.98 \pm 9.33$ & $35.91 \pm 4.76$ & $12.08 \pm 2.11$ \\
\hline 28 & $2,6-2 F$ & $174.17 \pm 17.26$ & $46.20 \pm 5.70$ & $25.3 I \pm 4.34$ & $46.25 \pm 5.72$ \\
\hline 29 & $3,4-2 F$ & $81.18 \pm 10.43$ & $38.46 \pm 4.65$ & $43.77 \pm 5.32$ & $50.73 \pm 6.73$ \\
\hline 30 & $2-\mathrm{F}, 4-\mathrm{Cl}$ & $150.48 \pm 13.26$ & $38.86 \pm 4.26$ & $14.32 \pm 1.93$ & $54.39 \pm 6.26$ \\
\hline 31 & $2-\mathrm{Br}, 4-\mathrm{F}$ & $59.58 \pm 6.83$ & $44.23 \pm 5.52$ & $18.88 \pm 2.36$ & $18.84 \pm 2.83$ \\
\hline 32 & $2,4-2 \mathrm{Cl}$ & $28.20 \pm 3.46$ & $28.09 \pm 2.68$ & $27.94 \pm 2.53$ & $65.96 \pm 7.26$ \\
\hline 33 & $2,5-2 \mathrm{Cl}$ & $65.50 \pm 7.22$ & $38.57 \pm 4.57$ & $24.21 \pm 3.48$ & $7.88 \pm 1.12$ \\
\hline 34 & $2,6-2 \mathrm{Cl}$ & $73.63 \pm 8.94$ & $22.76 \pm 2.26$ & $15.67 \pm 2.33$ & $20.91 \pm 2.90$ \\
\hline 35 & $3,4-2 \mathrm{Cl}$ & $30.78 \pm 4.32$ & $20.35 \pm 2.35$ & $16.15 \pm 2.92$ & $|5.68 \pm 2.3|$ \\
\hline 36 & $2,4,6-3 \mathrm{Cl}$ & $12.57 \pm 1.96$ & $13.32 \pm 2.36$ & $18.68 \pm 2.69$ & $30.47 \pm 3.47$ \\
\hline BBR & & $121.91 \pm 11.26$ & $27.64 \pm 3.04$ & $68.06 \pm 7.76$ & $|8.33 \pm 2.3|$ \\
\hline
\end{tabular}

Note: ${ }^{a} \mathrm{C}_{50}$ is the drug concentration effective in inhibiting $50 \%$ of the cell growth measured by the MTT assay after 48 hours of drug exposure.

Abbreviations: BBR, berberine; HUVEC, human umbilical vein endothelial cell line; $I_{50}$, half-maximal inhibitory concentration; MCF-7, human breast adenocarcinoma antidrug resistance cell line; MTT, yellow tetrazolium salt 3-(4,5-Dimethylthiazol-2-yl)-2,5-diphenyltetrazolium bromide; R, substituent on the benzene ring; SMMC-772I, human liver carcinoma cell line; SW-1990, human pancreatic carcinoma cell line. 


\section{Results and discussion}

\section{In vivo anticancer activity}

From the $\mathrm{IC}_{50}$ values (Table 1) and percentage inhibition data (Figures 2-4), it is obvious that most of the tested compounds displayed better anticancer activities than that of the reference drug berberine with an $\mathrm{IC}_{50}$ value of $121.91 \pm 11.26 \mu \mathrm{M}$ (MCF-7) and 68.06 $\pm 7.76 \mu \mathrm{M}$ (SMMC-7721). However, the activity of berberine against SW-1990 cells was better than that of the derivatives, with an $\mathrm{IC}_{50}$ value of $27.64 \pm 3.04 \mu \mathrm{M}$. Berberine only exhibited greater than $50 \%$ inhibition against SW-1990 cell growth at $62.5 \mu \mathrm{M}$. In addition, the tested compounds generally showed a lower effect on noncancer cells (HUVECs) than the reference drug berberine, which had an $\mathrm{IC}_{50}$ value of $18.33 \pm 2.31 \mu \mathrm{M}$. For example, compounds 4, 14, 15, 17, 18, 26 and 32 against HUVEC cell line had $\mathrm{IC}_{50}$ values of $71.92 \pm 9.04,67.69 \pm 8.95$, $145.71 \pm 19.01,61.66 \pm 6.78,71.18 \pm 9.03,74.62 \pm 9.21$ and $65.96 \pm 7.26$, respectively.

Compound 16 has a tert-butyl group substituted phenyl, which inhibited the human pancreatic carcinoma SW-1990 cell line and human liver carcinoma SMMC-7721 cell line, with $\mathrm{IC}_{50}$ values of $8.54 \pm 1.97 \mu \mathrm{M}$ and $11.87 \pm 1.83 \mu \mathrm{M}$, while it had a lower effect than berberine on noncancerous HUVEC cells, with an $\mathrm{IC}_{50}$ value of $25.49 \pm 3.24 \mu \mathrm{M}$. In comparison, the $\mathrm{IC}_{50}$ values of compound 16 against MCF-7, SW-1990, and SMMC-7721 were 8, 3, and 6 times lower than that of berberine. However, it was less potent than compound 36 against the human breast adenocarcinoma MCF-7 cell line, with an $\mathrm{IC}_{50}$ value of $15.80 \pm 2.14 \mu \mathrm{M}$. Figures 2-4 show that compound 16 exhibited greater than $50 \%$ inhibition of MCF-7, SW-1990, and SMMC-7721 cell growth at $7.8 \mu \mathrm{M}$.
Compound 36 has 1, 3, 5-trichloro substituents instead of an $\mathrm{R}$ position. It showed the best activity of all of the derivatives against the MCF-7 cell line, with an $\mathrm{IC}_{50}$ value of $12.57 \pm 1.96 \mu \mathrm{M}$. It was less potent than compound 16 against the SW-1990 cell line ( $\mathrm{IC}_{50}$ value of $\left.13.32 \pm 2.36 \mu \mathrm{M}\right)$ and the SMMC-7721 cell line $\left(\mathrm{IC}_{50}\right.$ value of $\left.18.68 \pm 2.69 \mu \mathrm{M}\right)$, and displayed lower cytotoxicity than berberine to HUVEC cells, with an $\mathrm{IC}_{50}$ value of $30.47 \pm 3.47 \mu \mathrm{M}$. Furthermore, compound 36 exhibited greater than $45 \%$ inhibition of MCF-7 cell growth at $7.8 \mu \mathrm{M}$ (Figure 2), which is much better than the reference drug berberine and other target compounds.

Compounds 4 ( $\mathrm{R}=-\mathrm{H}), 5-7(\mathrm{R}=-2-\mathrm{F},-3-\mathrm{F},-4-\mathrm{F}), 8-10$ $(\mathrm{R}=-2-\mathrm{Cl},-3-\mathrm{Cl},-4-\mathrm{Cl}), 11-13(\mathrm{R}=-2-\mathrm{Br},-3-\mathrm{Br},-4-\mathrm{Br})$, $14-15\left(\mathrm{R}=-2-\mathrm{CH}_{3},-4-\mathrm{CH}_{3}\right), 17-18\left(\mathrm{R}=-3-\mathrm{OCH}_{3},-4-\mathrm{OCH}_{3}\right)$, and $24\left(\mathrm{R}=-4-\mathrm{NO}_{2}\right)$ were single substitution at the $\mathrm{R}$ position. Among them, compound 14 inhibited the SMMC-7721 cell line, with an $\mathrm{IC}_{50}$ value of $19.95 \pm 2.33 \mu \mathrm{M}$. Compound 15 inhibited the MCF-7, SW-1990, and SMMC-7721 cell lines, with $\mathrm{IC}_{50}$ values of $37.83 \pm 4.66 \mu \mathrm{M}, 32.03 \pm 4.23 \mu \mathrm{M}$, and $39.37 \pm 4.98 \mu \mathrm{M}$, respectively. However, compound 24 exhibited weaker activities than compound 15 against the three cancer cell lines (MCF-7, SW-1990, SMMC-7721), with $\mathrm{IC}_{50}$ values of $206.47 \pm 23.59 \mu \mathrm{M}, 54.23 \pm 6.55 \mu \mathrm{M}$, and $63.81 \pm 8.90 \mu \mathrm{M}$, respectively. Compound 4 contained no group on the substituted phenyl ring and exhibited weaker anticancer activities against the three cancer cell lines than compound 15 but better activities than compound 13 . Compounds 5, 6, and 7 have a single fluorine instead of an aromatic ring at the ortho, meta, and para positions. However, these compounds showed weaker anticancer activities than

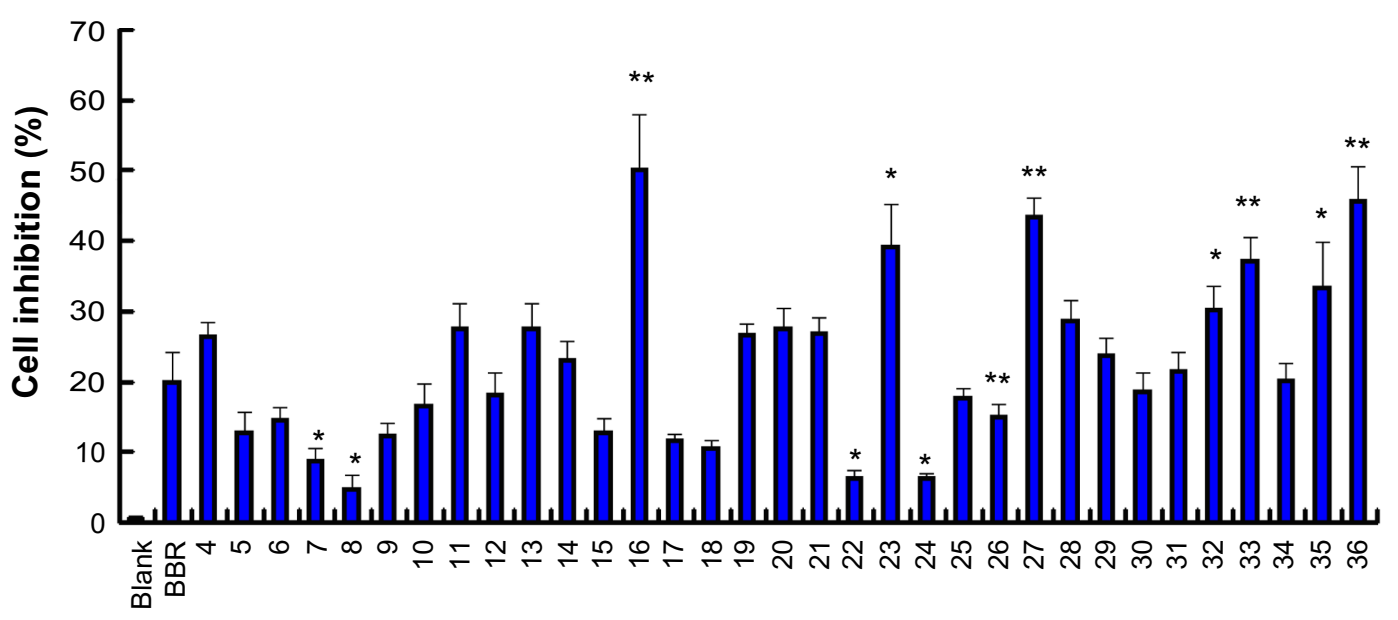

Figure 2 Inhibitory rate (\%) of compounds 4-36 against MCF-7 cell line at $7.8 \mu \mathrm{M}$.

Notes: Data are presented as mean \pm SD of at last three replicates from three independent experiments. $* P<0.05$ versus $B B R$. $* * P<0.0$ I versus $B B R$.

Abbreviations: BBR, berberine; Blank, culture medium alone, without compounds; MCF-7, human breast adenocarcinoma antidrug resistance cell line; SD, standard deviation. 


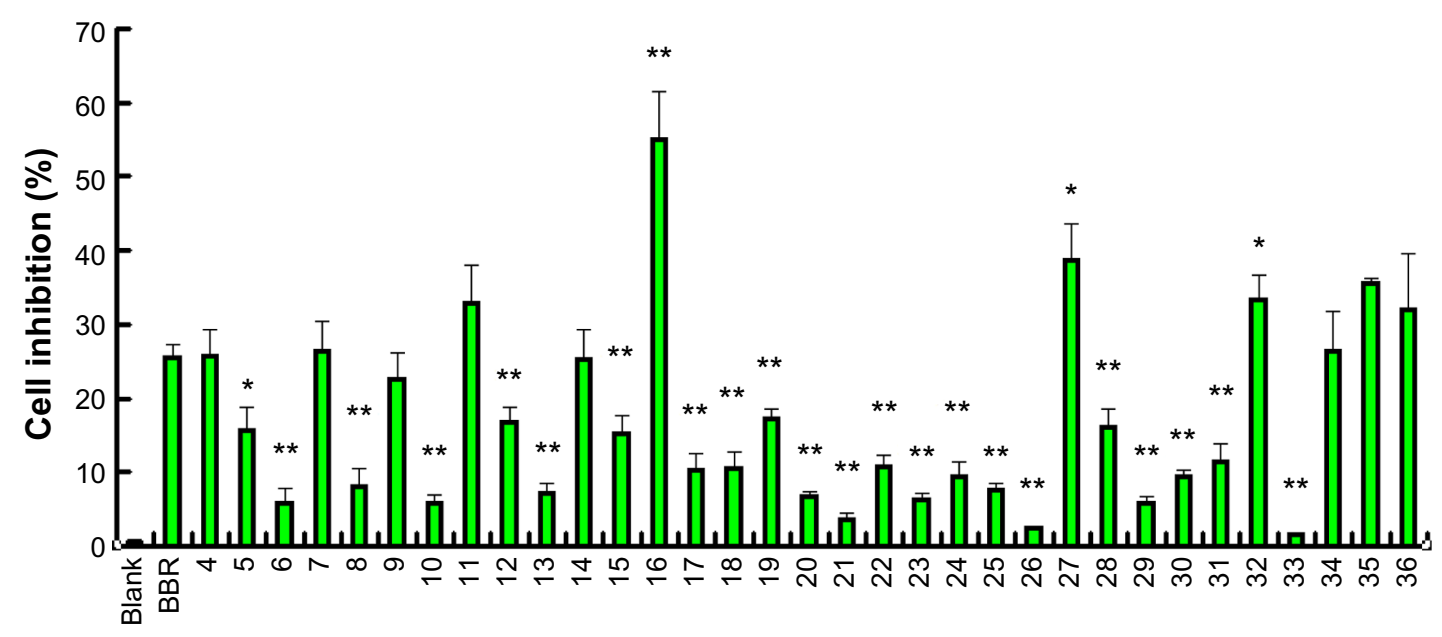

Figure 3 Inhibitory rate (\%) of compounds 4-36 against SW-1990 cell line at $7.8 \mu \mathrm{M}$.

Notes: Data are presented as mean \pm SD of at last three replicates from three independent experiments. $* P<0.05$ versus $B B R$; $* * P<0.01$ versus $B B R$.

Abbreviations: BBR, berberine; Blank, culture medium alone, without compounds; SD, standard deviation; SW-1990, human pancreatic carcinoma cell line.

any other compounds with electron-withdrawing substituents on the phenyl group (compounds 8-13).

Among the derivatives tested, compounds 25 and 26 had 4-methyl formate and 4-methylsulfonyl at the $\mathrm{R}$ position, respectively, which inhibited against four cancer cells lines with $\mathrm{IC}_{50}$ values of $248.55 \pm 20.13 \mu \mathrm{M}$ (MCF-7), 165.27 $\pm 18.94 \mu \mathrm{M}$ (SW-1990), and 53.49 $\pm 5.84 \mu \mathrm{M}$ (SMMC-7721), and 206.84 $\pm 19.65 \mu \mathrm{M}$ (MCF-7), 100.76 $\pm 9.01 \mu \mathrm{M}$ (SW-1990), and 69.68 $\pm 8.93 \mu \mathrm{M}$ (SMMC-7721), respectively.

From the data, we can indicate that adding disubstituted groups on the phenyl ring of compounds 27-35 showed moderate effect against three human cancer cell lines. However, compounds 32-35 had a dichlorine group at the $\mathrm{R}$ position and had enhanced activity compared to that of other electron-withdrawing group derivatives (compounds 27-31). Compounds 32 and 35 had 2, 4-dichloro and 3, 4-dichloro substituents on the phenyl group, respectively. Compounds 32 and 35 inhibited against the MCF-7, SW-1990, and SMMC-7721 cell lines, with $\mathrm{IC}_{50}$ values of $28.20 \pm 3.46 \mu \mathrm{M}$ (compound 32, MCF7), $30.78 \pm 4.32 \mu \mathrm{M}$ (compound 35, MCF-7), 28.09 $\pm 2.68 \mu \mathrm{M}$ (compound 32, SW-1990), 20.35 $\pm 2.35 \mu \mathrm{M}$ (compound 35, SW-1990), 27.94 $\pm 2.53 \mu \mathrm{M}$ (compound 32, SMMC-7721), and $16.15 \pm 2.92 \mu \mathrm{M}$ (compound 35, SMMC-7721). However, the $\mathrm{IC}_{50}$ value of compound 35 against HUVECs was four times lower than that of 32 . It is obvious that compound 32 showed weaker cytotoxicity than compound 35 against the noncancer HUVEC cell line. Besides, among these derivatives, compound 33 showed the most potent cytotoxicity against HUVECs, with an $\mathrm{IC}_{50}$ value of $7.88 \pm 1.12 \mu \mathrm{M}$.

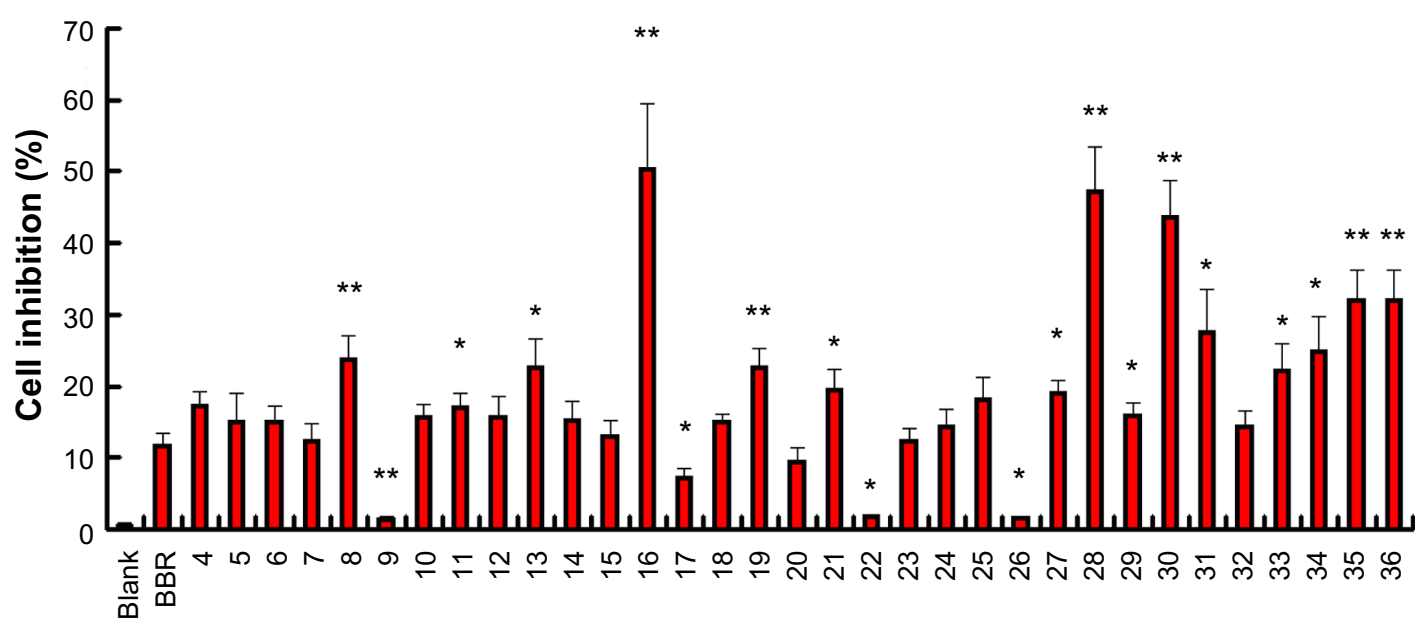

Figure 4 Inhibitory rate (\%) of compounds 4-36 against SMMC-772I cell line at 7.8 $\mu$ M.

Notes: Data are presented as mean \pm SD of at last three replicates from three independent experiments. $* P<0.05$ versus $B B R$. $* * P<0.0$ I versus $B B R$. Abbreviations: BBR, berberine; Blank, culture medium alone, without compounds; SD, standard deviation; SMMC-772I, human liver carcinoma cell line. 
Compared to compound $32(\mathrm{R}=-2,4-2 \mathrm{Cl})$ the activity of compound 30 ( $\mathrm{R}=-2-\mathrm{F}, 4-\mathrm{Cl})$ was weaker. Similarly, compound 27 had 2-4-difluorine substituents on the phenyl group, but the cytotoxicity of compound 27 against the three cancer cell lines was lower than that of compound 31 $(\mathrm{R}=-2-\mathrm{Br}, 4-\mathrm{F})$.

\section{Conclusions}

This study described the construction of a new series of triazole-containing berberine derivatives using $\mathrm{CuAAC}$ "click" chemistry. All of the compounds synthesized were screened for anticancer activity against a panel of three human cancer cell lines, MCF-7 (breast), SW-1990 (pancreatic), and SMMC-7721 (liver), and the noncancer HUVEC (human umbilical vein endothelial cell) cell line. From the data, it was evident that most of the target compounds exhibited better anticancer activity against all the tested cancer cell lines than the reference drug berberine used in this study. Compounds 16 and 36, which had tert-butyl and 1,3,5-trichlorosubstituents on the phenyl group, were the most promising target compounds. It was noted that highly active anticancer compounds showed lower cytotoxicity to the noncancerous HUVEC cells compared with berberine. Detailed mechanistic studies are currently underway in our laboratory.

\section{Acknowledgments}

This work was supported by the National Natural Science Foundation of China (General Program No 81273556/ H3106) and by Shanghai Key Projects of Basic Research (Number 11JC1415400).

\section{Disclosure}

The authors report no conflicts of interest in this work.

\section{References}

1. Ciardiello F, Tortora G. A novel approach in the treatment of cancer: targeting the epidermal growth factor receptor. Clin Cancer Res. 2001:7(10):2958-2970.

2. Brannon-Peppas L, Blanchette JO. Nanoparticle and targeted systems for cancer therapy. Adv Drug Deliv Rev. 2004;56(11):1649-1659.

3. Aird RE, Cummings J, Ritchie AA, et al. In vitro and in vivo activity and cross resistance profiles of novel ruthenium (II) organometallic arene complexes in human ovarian cancer. Br J Cancer. 2002;86(10): 1652-1657.

4. Grycová L, Dostál J, Marek R. Quaternary protoberberine alkaloids. Phytochemistry. 2007;68(2):150-175.

5. Iwasa K, Kamigauchi M, Ueki M, Taniguchi M. Antibacterial activity and structure-activity relationships of berberine analogs. Eur J Med Chem. 1996;31(6):469-478.

6. Iwasa K, Kim HS, Wataya Y, Lee DU. Antimalarial activity and structure-activity relationships of protoberberine alkaloids. Eur J Med Chem. 1998;33(1):65-69.
7. Vennerstrom JL, Lovelace JK, Waits VB, Hanson WL, Klayman DL. Berberine derivatives as antileishmanial drugs. Antimicrob Agents Chemother. 1990;34(5):918-921.

8. Yang P, Song DQ, Li YH, et al. Synthesis and structure-activity relationships of berberine analogues as a novel class of low-densitylipoprotein receptor up-regulators. Bioorg Med Chem Lett. 2008; 18(16):4675-4677

9. Samosorn S, Tanwirat B, Muhamad N, et al. Antibacterial activity of berberine-NorA pump inhibitor hybrids with a methylene ether linking group. Bioorg Med Chem. 2009;17(11):3866-3872.

10. Park KD, Lee JH, Kim SH, Kang TH, Moon JS, Kim SU. Synthesis of 13-(substituted benzyl) berberine and berberrubine derivatives as antifungal agents. Bioorg Med Chem Lett. 2006;16(15): 3913-3916.

11. Chen WH, Pang JY, Qin Y, Peng Q, Cai Z, Jiang ZH. Synthesis of linked berberine dimers and their remarkably enhanced DNA-binding affinities. Bioorg Med Chem Lett. 2005;15(10):2689-2692.

12. Hoshi A, Ikekawa T, Ikeda Y, Shirakawa S, Iigo M. Antitumor activity of berberrubine derivatives. Gann. 1976;67(2):321-325.

13. Lo CY, Hsu LC, Chen MS, et al. Synthesis and anticancer activity of a novel series of 9-O-substituted berberine derivatives: a lipophilic substitute role. Bioorg Med Chem Lett. 2013;23(1):305-309.

14. Pang JY, Qin Y, Chen WH, Luo GA, Jiang ZH. Synthesis and DNA-binding affinities of monomodified berberines. Bioorg Med Chem. 2005;13(20):5835-5840.

15. Zhang WJ, Ou TM, Lu YJ, et al. 9-Substituted berberine derivatives as G-quadruplex stabilizing ligands in telomeric DNA. Bioorg Med Chem. 2007;15(16):5493-5501.

16. Ma Y, Ou TM, Hou JQ, et al. 9-N-Substituted berberine derivatives: stabilization of G-quadruplex DNA and down-regulation of oncogene c-myc. Bioorg Med Chem. 2008;16(16):7582-7591.

17. Li QY, Zu YG, Liu TY, et al. Generation of reactive oxygen species by a novel berberine-bile acid analog mediates apoptosis in hepatocarcinoma SMMC-7721 cells. Biochem Biophys Res Commun. 2013;433(4):432-437.

18. Franceschin M, Rossetti L, D’Ambrosio A, et al. Natural and synthetic G-quadruplex interactive berberine derivatives. Bioorg Med Chem Lett. 2006;16(6):1707-1711.

19. Beausoleil E, Chauvignac C, Taverne T, et al. Structure-activity relationship of isoform selective inhibitors of Rac1/1b GTPase nucleotide binding. Bioorg Med Chem Lett. 2009;19(19):5594-5598.

20. Pan GY, Wang GJ, Liu XD, Fawcett JP, Xie YY. The involvement of P-glycoprotein in berberine absorption. Pharmacol Toxicol. 2002;91(4): 193-197.

21. Chen CM, Chang HC. Determination of berberine in plasma, urine and bile by high-performance liquid chromatography. J Chromatogr B Biomed Appl. 1995;665(1):117-123.

22. Kolb HC, Finn MG, Sharpless KB. Click chemistry: diverse chemical function from a few good reactions. Angew Chem Int Ed Engl. 2001;40(11):2004-2021.

23. Lutz JF; Nanotechnology for Life Science Research Group. 1,3-dipolar cycloadditions of azides and alkynes: a universal ligation tool in polymer and materials science. Angew Chem Int Ed Engl. 2007;46(7): 1018-1025.

24. Rostovtsev VV, Green LG, Fokin VV, Sharpless KB. A stepwise huisgen cycloaddition process: copper(I)-catalyzed regioselective "ligation" of azides and terminal alkynes. Angew Chem Int Ed Engl. 2002;41(14):2596-2599.

25. Tornøe CW, Christensen C, Meldal M. Peptidotriazoles on solid phase: [1,2,3]-triazoles by regiospecific copper(i)-catalyzed 1,3dipolar cycloadditions of terminal alkynes to azides. $\mathrm{J}$ Org Chem. 2002;67(9):3057-3064.

26. Binder WH, Sachsenhofer R. "Click" chemistry in polymer and materials science. Macromol Rapid Commun. 2007;28(1):15-54.

27. Bock VD, Hiemstra $\mathrm{H}$, van Maarseveen JH. Cu -catalyzed alkyne-azide "click" cycloadditions from a mechanistic and synthetic perspective. Eur J Org Chem. 2006;2006(1):51-68. 
28. Fournier D, Hoogenboom R, Schubert US. Clicking polymers: a straightforward approach to novel macromolecular architectures. Chem Soc Rev. 2007;36(8):1369-1380.

29. Angell YL, Burgess K. Peptidomimetics via copper-catalyzed azidealkyne cycloadditions. Chem Soc Rev. 2007;36(10):1674-1689.

30. Wang Q, Chittaboina S, Barnhill HN. Highlights in organic chemistry advances in 1,3-dipolar cycloaddition reaction of azides and alkynes a prototype of "click" chemistry. Lett Org Chem. 2005;2(4):293-301.

31. Hua Y, Flood AH. Click chemistry generates privileged $\mathrm{CH}$ hydrogenbonding triazoles: the latest addition to anion supramolecular chemistry. Chem Soc Rev. 2010;39(4):1262-1271.
32. Nandivada H, Jiang $X$, Lahann J. Click chemistry: versatility and control in the hands of materials scientists. Adv Mat. 2007;19(17): 2197-2208.

33. Bodiwala HS, Sabde S, Mitra D, Bhutani KK, Singh IP. Synthesis of 9-substituted derivatives of berberine as anti-HIV agents. Eur J Med Chem. 2011;46(4):1045-1049.

34. Sieuwerts AM, Klijn JG, Peters HA, Foekens JA. The MTT tetrazolium salt assay scrutinized: how to use this assay reliably to measure metabolic activity of cell cultures in vitro for the assessment of growth characteristics, IC50-values and cell survival. Eur J Clin Chem Clin Biochem. 1995;33(11):813-823.

\section{Publish your work in this journal}

Drug Design, Development and Therapy is an international, peerreviewed open-access journal that spans the spectrum of drug design and development through to clinical applications. Clinical outcomes, patient safety, and programs for the development and effective, safe, and sustained use of medicines are a feature of the journal, which has also been accepted for indexing on PubMed Central. The manuscript management system is completely online and includes a very quick and fair peer-review system, which is all easy to use. Visit http://www.dovepress.com/testimonials.php to read real quotes from published authors.

Submit your manuscript here: http://www.dovepress.com/drug-design-development-and-therapy-journal 